\title{
The nature of UCDs: Internal dynamics from an expanded sample and homogeneous database ${ }^{\star, \star \star}$
}

\author{
S. Mieske ${ }^{1}$, M. Hilker ${ }^{1}$, A. Jordán ${ }^{2,3}$, L. Infante ${ }^{3}$, M. Kissler-Patig ${ }^{1}$, M. Rejkuba ${ }^{1}$, T. Richtler ${ }^{4}$, \\ P. Côté ${ }^{5}$, H. Baumgardt ${ }^{6}$, M. J. West ${ }^{7}$, L. Ferrarese ${ }^{5}$, and E. W. Peng ${ }^{5}$ \\ ${ }^{1}$ European Southern Observatory, Karl-Schwarzschild-Strasse 2, 85748 Garching bei München, Germany \\ e-mail: smieske@eso.org \\ 2 Clay Fellow, Harvard-Smithsonian Center for Astrophysics, 60 Garden St., Cambridge, MA 02138, USA \\ 3 Departamento de Astronomía y Astrofísica, Pontificia Universidad Católica de Chile, Casilla 306, Santiago 22, Chile \\ ${ }^{4}$ Universidad de Concepción, Departamento de Fisica, Astronomy Group, Casilla 160-C, Concepción, Chile \\ 5 National Research Council of Canada, Herzberg Institute of Astrophysics, 5071 West Saanich Road, Victoria, BC V9E 2E7, Canada \\ 6 Argelander-Institut für Astronomie, Auf dem Hügel 71, 53121 Bonn, Germany \\ 7 European Southern Observatory, Alonso de Córdova 3107, Vitacura, Casilla 19001, Santiago, Chile
}

Received 28 April 2008 / Accepted 23 May 2008

ABSTRACT

Context. The internal dynamics of ultra-compact dwarf galaxies (UCDs) has attracted increasing attention, with most of the UCDs studied to date located in the Virgo cluster.

Aims. Our aim is to perform a comprehensive census of the internal dynamics of UCDs in the Fornax cluster, and to shed light on the nature of the interface between star clusters and galaxies.

Methods. We obtained high-resolution spectra of 23 Fornax UCDs with $-10.4>M_{V}>-13.5 \operatorname{mag}\left(10^{6}<M / M_{\odot}<10^{8}\right)$, using FLAMES/Giraffe at the VLT. This is the largest homogeneous data set of UCD internal dynamics assembled to date. We derive dynamical $M / L$ ratios for 15 UCDs covered by HST imaging.

Results. In the $M_{V}-\sigma$ plane, UCDs with $M_{V}<-12$ mag are consistent with the extrapolated Faber-Jackson relation for luminous elliptical galaxies, while most of the fainter UCDs are closer to the extrapolated globular cluster (GC) relation. At a given metallicity, Fornax UCDs have, on average, $M / L$ ratios lower by $30-40 \%$ than Virgo UCDs, suggesting possible differences in age or dark matter content between Fornax and Virgo UCDs. For our sample of Fornax UCDs we find no significant correlation between $M / L$ ratio and mass. We combine our data with available $M / L$ ratio measurements of compact stellar systems with $10^{4}<M / M_{\odot}<10^{8} \mathrm{M}$, and normalise all $M / L$ estimates to solar metallicity. We find that UCDs $\left(M \gtrsim 2 \times 10^{6} M_{\odot}\right)$ have $M / L$ ratios twice as large as GCs $\left(M \lesssim 2 \times 10^{6} M_{\odot}\right)$. We argue that dynamical evolution has probably had only a small effect on the current $M / L$ ratios of objects in the combined sample, implying that stellar population models tend to under-predict dynamical $M / L$ ratios of UCDs and over-predict those of GCs. Considering the scaling relations of stellar spheroids, we find that UCDs align well along the "Fundamental Manifold". UCDs can be considered the small-scale end of the galaxy sequence in this context. The alignment for UCDs is especially clear for $r_{\mathrm{e}} \gtrsim 7 \mathrm{pc}$, which corresponds to dynamical relaxation times that exceed a Hubble time. In contrast, globular clusters exhibit a broader scatter and do not appear to align along the manifold.

Conclusions. We argue that UCDs are the smallest dynamically un-relaxed stellar systems, with $M \gtrsim 2 \times 10^{6} M_{\odot}$ and $7 \lesssim r_{\mathrm{e}} / \mathrm{pc} \lesssim 100$. Future studies should aim at explaining the elevated $M / L$ ratios of UCDs and the environmental dependence of their properties.

Key words. galaxies: clusters: individual: Fornax - galaxies: dwarf - galaxies: fundamental parameters - galaxies: nuclei galaxies: star clusters

\section{Introduction}

In recent years, significant effort has been devoted to studying the internal dynamics of extragalactic compact stellar systems in the mass regime of massive globular clusters and ultra-compact dwarf galaxies $\left(10^{6}<M / M_{\odot}<10^{8}\right)$ (Drinkwater et al. 2003; Martini \& Ho 2004; Haşegan et al. 2005; Maraston et al. 2004; Rejkuba et al. 2007; Evstigneeva et al. 2007; Hilker et al. 2007). A compilation of the available data is presented in Mieske \& Kroupa (2008) and Dabringhausen et al. (2008).

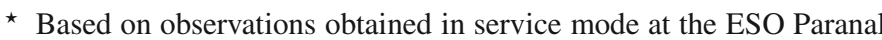
Observatory with the VLT (programme 078.B-0496).

$\star \star$ Table 5 is only available in electronic form at

http://www . aanda.org
}

A striking outcome of these studies is the finding that the dynamical $M / L$ ratios of massive compact stellar systems are, on average, about two times larger than those of normal globular clusters of comparable metallicity. Several objects have $M / L$ ratios at the limit of, or even beyond, the range predicted by stellar population models assuming canonical IMFs (Haşegan et al. 2005). Possible explanations for these high $M / L$ ratios include extreme stellar mass functions (Mieske \& Kroupa 2008; Dabringhausen et al. 2008) or densely packed dark matter (Goerdt et al. 2008). The occurence of objects with high $M / L$ ratios is observed to start at $\sim 2 \times 10^{6} M_{\odot}$ (Haşegan et al. 2005; Rejkuba et al. 2007; Mieske \& Kroupa 2008), coinciding with "breaks" in physical size (Haşegan et al. 2005; Mieske et al. 2006; Kissler-Patig et al. 2006) and stellar content (Mieske et al. 2006). These breaks are consistent with the hypothesis that at 
$\sim 2 \times 10^{6} M_{\odot}\left(M_{V} \simeq-11 \mathrm{mag}\right)$ we observe the transition between simple globular clusters and more complex systems, the UCDs. UCDs:

Three main formation scenarios have been suggested for

1. UCDs are stellar super clusters formed in the tidal arms of violent gas-rich galaxy mergers (Fellhauer \& Kroupa 2002, 2005).

2. UCDs are tidally stripped compact remnants of nucleated dwarf galaxies (Bassino et al. 1994; Hilker et al. 1999; Bekki et al. 2003; Goerdt et al. 2008).

3. UCDs are genuine compact dwarf galaxies formed from small-scale peaks in the primordial dark matter power spectrum (Drinkwater et al. 2004).

In the first case, UCDs are not expected to contain any dark matter. In the second and third case, UCDs would be related to cosmological low-mass dark matter halos and may contain dark matter (Goerdt et al. 2008). The comparably large $M / L$ ratios of 5-10 found for some Virgo UCDs may point towards a cosmological origin. These $M / L$ ratios are similar to the values found for some of the more luminous Local Group dSphs like Sculptor and LeoI (Gilmore et al. 2007), although note that they are still 1-2 orders of magnitude below the $M / L$ values found for the ultra-faint dSph candidates (Gilmore et al. 2007; Simon \& Geha 2007). In this paper, we aim to study whether the high $M / L$ ratios are a fundamental trend equally common to all UCDs, or whether environmental variations of the trend exist. The latter may be expected if competing formation channels dominate in different environments (e.g. Mieske et al. 2006). For both the Virgo cluster (Haşegan et al. 2005; Evstigneeva et al. 2007) and the Centaurus A group (Rejkuba et al. 2007), more than 10 objects in the UCD mass range have measured $M / L$ ratios. For the Fornax cluster, only five sources have high resolution spectroscopy available (Hilker et al. 2007), making it difficult to judge whether differences exist between Fornax and Virgo UCDs. With a comprehensive sample of measurements for Fornax, it will be possible to analyse whether Fornax UCDs extend to such high $M / L$ ratios as Virgo UCDs.

In what follows, we present new measurements of the internal kinematics of 23 compact objects in Fornax (obtained with FLAMES/GIRAFFE at the VLT). We analyse how their $M / L$ ratios relate to predictions from stellar population models, and investigate how they fit into the trend of increasing $M / L$ with mass among compact stellar systems. We also examine how UCDs and globular clusters fit into the broader context of larger and more luminous stellar systems, focusing on the fundamental manifold of stellar spheroids (Zaritsky et al. 2006a,b, 2008) that is an extension of the fundamental plane concept.

The paper is structured as follows: in Sect. 2 we present the new spectroscopic data of Fornax UCDs used for the present study. Section 3 describes the data reduction, including the modelling of the mass distribution. In Sect. 4, the results for the Fornax UCDs are presented and discussed. In Sect. 5 we combine the Fornax data with other literature results on dynamical $\mathrm{M} / \mathrm{L}$ ratios of compact stellar systems, and investigate UCDs and GCs in the context of the fundamental manifold. The paper finishes with Summary and Conclusions in Sect. 6. Throughout this paper we assume a distance modulus to Fornax of $(m-M)=$ 31.4 mag (Freedman et al. 2001).

\section{Data}

The data for this study were obtained in service mode with the Fibre Large Array Multi Element Spectrograph (FLAMES; Pasquini et al. 2002) mounted on UT2 at the VLT (programme 078.B-0496). We used the spectrograph GIRAFFE in MEDUSA mode, which allows the observation of up to 130 targets at the same time over a 25 arcmin diameter field of view, using fibres of $1.2^{\prime \prime}$ aperture. We observed a total of $15 \mathrm{~h}$ onsource, subdivided in 15 individual integrations of $1 \mathrm{~h}$ duration.

Figure 1 shows a map of the observed region. We observed 37 compact objects within $12^{\prime}$ of NGC 1399 and with $18<$ $V<21$ mag $\left(-13.4<M_{V}<-10.4 \mathrm{mag}\right)$. This magnitude range covers the UCDs and overlaps the bright end of the globular cluster luminosity function (Mieske et al. 2004). All targets have confirmed cluster membership from spectroscopic surveys (Drinkwater et al. 2000; Mieske et al. 2002, 2004; Richtler et al. 2004, 2008), except for the two objects closest to NGC 1399 , which were selected on the basis of their morphology from imaging from the ACS Fornax cluster survey (Jordán et al. 2007).

For the observations we used the HR09A grism, which provides an instrumental resolution of $8 \mathrm{~km} \mathrm{~s}^{-1}$ in terms of Gaussian $\sigma$ (or $19 \mathrm{~km} \mathrm{~s}^{-1}$ in terms of FWHM) over a wavelength range $5100<\lambda<5400 \AA$. This resolution allows us to reliably measure velocity dispersions $\geq 10 \mathrm{~km} \mathrm{~s}^{-1}$.

\section{Data reduction}

\subsection{Basic reduction}

In order to remove the instrumental signatures from the data, we used the publicly available GIRAFFE data reduction pipeline from $\mathrm{ESO}^{1}$

This pipeline performs bias subtraction, flat-field division, wavelength calibration, and spectrum extraction. As such, it creates a wavelength calibrated 1D spectrum from a raw 2D spectrum. The pixel scale in the wavelength calibrated 1D spectrum is $0.05 \AA$ per pixel, slightly over-sampling the instrumental scale of $0.08 \AA$ per pixel. The rms of the wavelength solution was of the order $0.1 \AA$. The instrumental resolution - resulting from the four pixel FWHM of the fiber's spatial profile - in terms of Gaussian $\sigma$ is $\sim 0.14 \AA$. This instrumental resolution corresponds to a velocity dispersion of $\sigma \sim 8 \mathrm{~km} \mathrm{~s}^{-1}$ in the $5100-5400 \AA$ wavelength regime.

Given the multiplexing capability of FLAMES/GIRAFFE, we also obtained 23 sky spectra in each exposure by assigning unoccupied fibres to empty sky positions. These spectra were reduced identically to the science spectra. We combined the 23 sky spectra in each exposure to 1 master sky spectrum, using the IRAF task scombine in the ONEDSPEC package. This combined sky spectrum was subtracted from each single, calibrated $1 \mathrm{D}$ object spectrum.

From this we obtained 15 sky-subtracted, calibrated 1D spectra for each of the 37 compact objects observed. Those single spectra were corrected to heliocentric velocity using the IRAF tasks rvcorrect in the RV package and dopcor in the ONEDSPEC package. The velocity shift between the 15 individual spectra due to shifts in wavelength calibration was very small $\left(<2 \mathrm{~km} \mathrm{~s}^{-1}\right)$. We combined the 15 registered single spectra using the IRAF task scombine. For this we normalised the

\footnotetext{
1 http://www . eso.org/projects/dfs/dfs-shared/web/vlt/ vlt-instrument-pipelines.html
} 

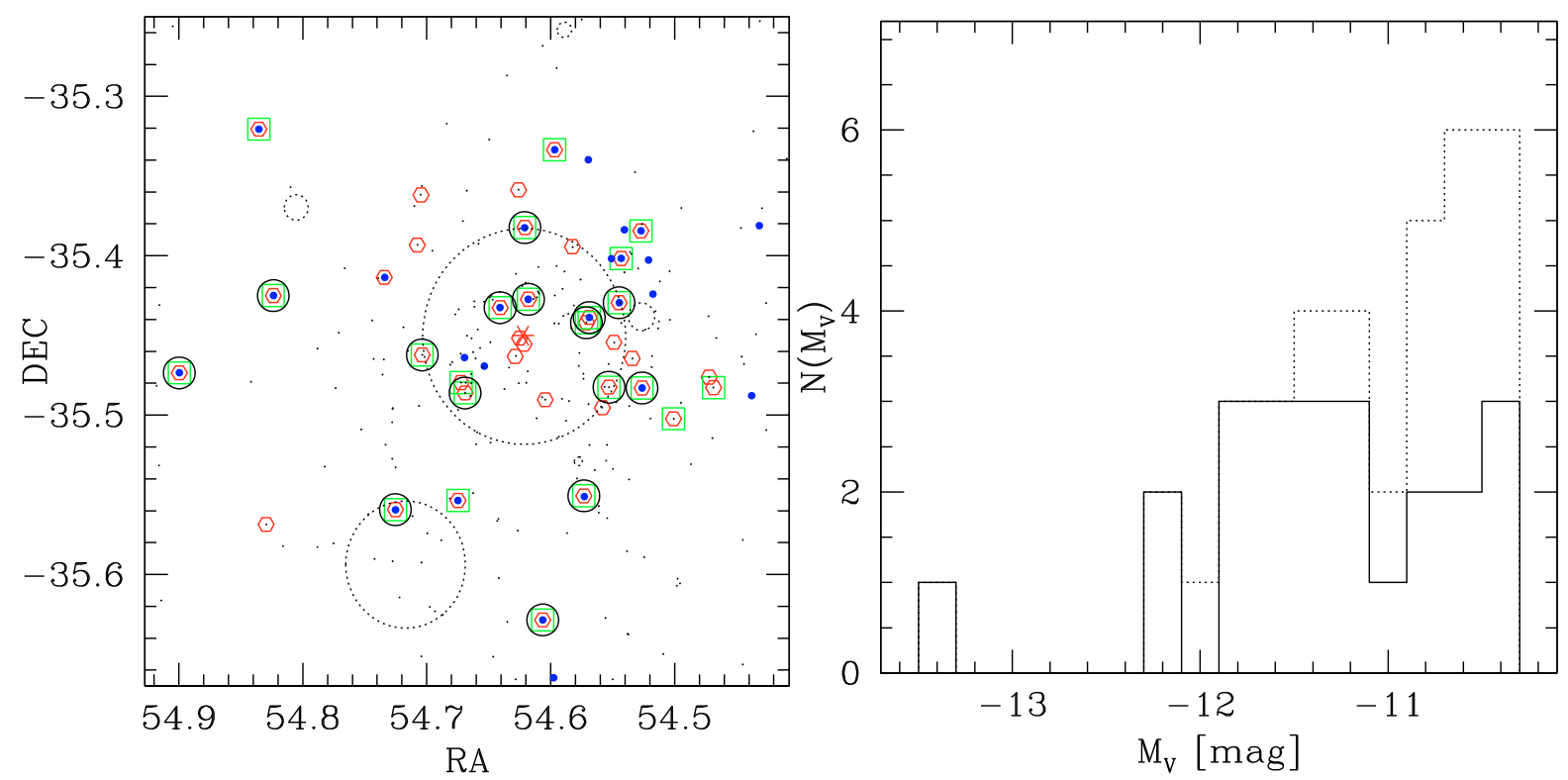

Fig. 1. Left: map of the observed region in the Fornax cluster. Hexagons indicate compact objects observed with FLAMES. Green squares indicate objects for which reliable velocity dispersions were measured. Large circles show targets with HST imaging that were successfully observed with FLAMES. Small dots are all known compact cluster members with $V<22$ mag $\left(M_{V}<-9.4\right.$ mag), large dots are those with $M_{V}<-11$ mag, the approximate magnitude division between UCDs and GCs. The dotted circles indicate Fornax cluster member galaxies from the Fornax Cluster Catalog (FCC; Ferguson 1989), for which the circle size gives the radius at which $\mu_{V}=25 \mathrm{mag} / \mathrm{arcsec}^{2}$. The asterisk marks the location of NGC 1399. Right: magnitude distribution of observed sources. The solid histogram refers to those objects for which reliable velocity dispersions were measured. The dotted histogram refers to all sources included in the fibre configuration (see the left panel).

intensity of the spectra to their mode and applied a $3.5 \sigma$ average sigma clipping algorithm. The resulting $S / N$ per pixel in the combined object spectra ranged between 5 and 35 .

In order to have template spectra for measuring the internal velocity dispersion, we also observed several dozen red giant stars in the Milky Way globular cluster $\omega$ Centauri in a single FLAMES/GIRAFFE pointing. These stars are of late spectral type (typical temperature $5000 \mathrm{~K}$ ), cover a metallicity range $-2.3<[\mathrm{Fe} / \mathrm{H}]<-0.5 \mathrm{dex}$ and have magnitudes around $V=$ 12 mag (van Loon et al. 2007). We used the same instrument setting and reduction procedures as for the science targets. The internal line width of the giant stars is negligible compared to the instrumental resolution. With $5 \mathrm{~min}$ on-source integration, we reached $S / N$ ratios between 50 and 100 . We used the 14 highest quality spectra as templates for the dispersion measurements.

In Fig. 2 we show examples of two object spectra and one template spectrum.

\subsection{Dispersion measurements}

The internal velocity dispersion, $\sigma$, of each compact object was measured by cross-correlating its spectrum with various template spectra (IRAF task fxcor; Tonry \& Davis 1979). For this measurement we excluded the wavelength region around the very strong Mgb lines $\left(\lambda_{\text {restframe }}<5210 \AA\right)$, since the measured width in this region proved to be systematically larger than in the rest of the spectra (see Fig. 3). Such an increased width in the very deep $\alpha$ element absorption features is likely caused by saturation effects, and has been found previously by other authors (e.g., Rejkuba et al. 2007; Hilker et al. 2007), who also excluded this region from their measurements. We used the wavelength region $5210<\lambda_{\text {restframe }}<5390 \AA$, which includes the many prominent Fe absorption features around $5325 \AA$ (see Fig. 2).

Prior to cross-correlation, we continuum subtracted the spectra. For this, we adjusted the continuum fitting order individually

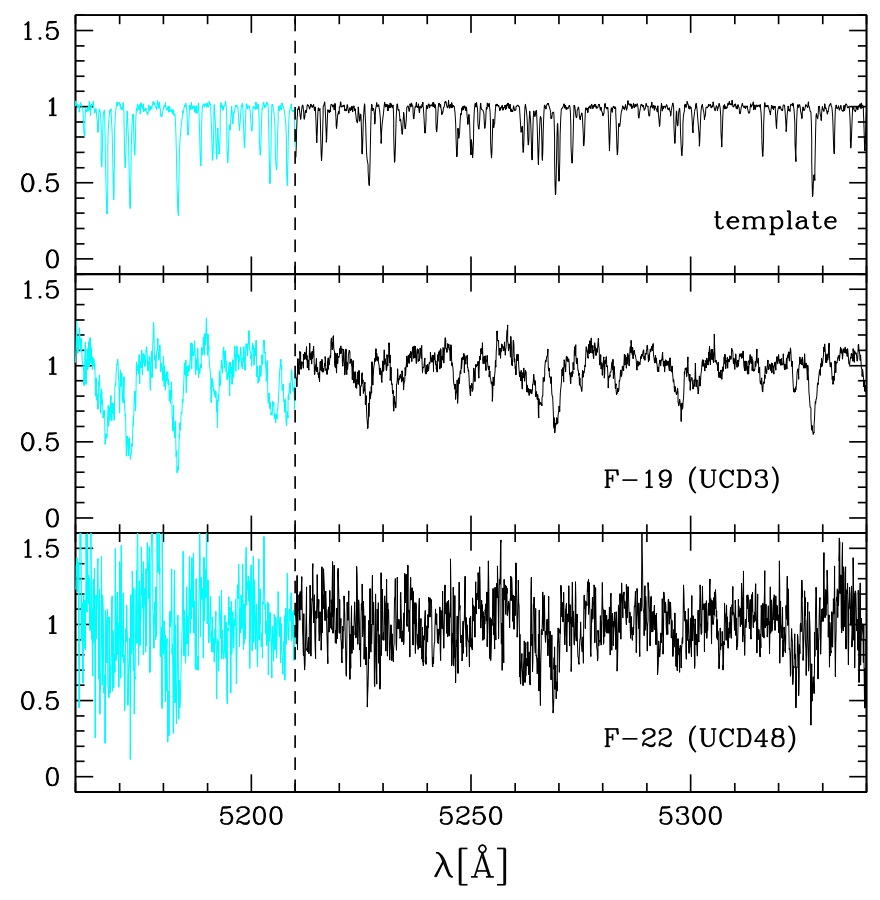

Fig. 2. Continuum normalised spectra of one template and two science objects, shifted to the restframe. The wavelength region $\lambda_{\text {restframe }}<$ $5210 \AA$ containing the $\mathrm{Mg}$ features was excluded from the crosscorrelation fit (see Fig. 3 and text).

for each source such as to yield the lowest order that gives satisfactory results. The peak position of the cross-correlation gives the relative radial velocity between object and template. The width, $\sigma_{\text {peak }}$, of the cross-correlation peak (Fig. 4) is the quadratic sum of the intrinsic object line width caused by random stellar motion plus twice the instrumental line width (equal 


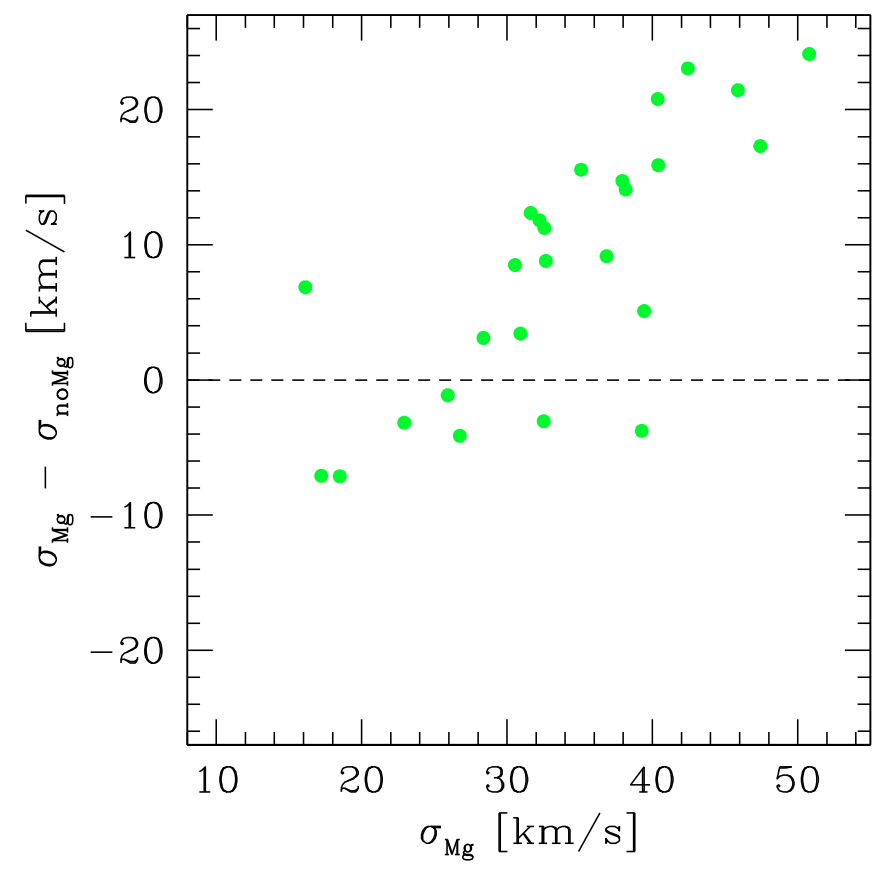

Fig. 3. The $x$-axis shows the Gaussian width $\sigma_{\mathrm{Mg}}$ in $\mathrm{km} \mathrm{s}^{-1}$ of the crosscorrelation peak derived in the $\mathrm{Mg}$ region $\left(5170<\lambda_{\text {restframe }}<5210 \AA\right)$. The $y$-axis shows the difference between this width and $\sigma$ derived in the rest of the spectrum $\left(5210<\lambda_{\text {restframe }}<5390 \AA\right)$. For $\sigma_{\mathrm{Mg}} \gtrsim 30 \mathrm{~km} \mathrm{~s}^{-1}$, the cross correlation width in the $\mathrm{Mg}$ region is significantly broader than in the rest of the spectrum.

to the template line width): i.e., $\sigma_{\text {peak }}^{2}=\sigma_{\text {obj }}^{2}+2 \times \sigma_{\text {ins }}^{2}$. By cross-correlating the un-broadened and continuum subtracted templates against each other, we measured the template's intrinsic line width $\sigma_{\text {ins }}$ to be $9.7 \mathrm{~km} \mathrm{~s}^{-1}$ with a very small scatter of order $0.4 \mathrm{~km} \mathrm{~s}^{-1}$. The intrinsic line width $\sigma_{\text {obj }}$ of the object spectrum is then calculated as: $\sigma_{\text {obj }}=\sqrt{\sigma_{\text {peak }}^{2}-2 \times \sigma_{\text {ins }}^{2}}$. Note that the factor 2 in front of $\sigma_{\text {ins }}^{2}$ is necessary because both the object and template spectrum are broadened by the instrumental resolution (Dubath et al. 1992). In Fig. 4, we show the cross-correlation results from the two objects whose spectra are shown in Fig. 2.

We performed tests with artificially broadened template spectra and different low frequency Fourier filter cutoffs to assess the accuracy of the fxcor task in measuring $\sigma_{\text {peak }}$. We found a slightly non-linear relation between input spectral width and width measured by fxcor. The best agreement was found for a low-frequency cutoff of $k=3$ (see Fig. 5). We adopted this cut-off for the Fourier filtering, and applied a residual correction as a linear function of $\sigma_{\mathrm{obj}}-$ as indicated in Fig. 5 - to the measured width of the science spectra. The residual correction is independent of the $S / N$ in the object spectra, which we tested by artificially degrading the broadened template spectra to a range of $S / N$ values between 5 and 35 per pixel, representative for our compact object sample. From the tests with the template spectra we also found that the background value in the cross-correlation peak fit needs to be kept fixed at 0 (see also Fig. 4). Allowing the program to fit the background value led to consistently overestimated widths.

We accepted a reliable measurement of $\sigma_{\text {obj }}$ for a given object if two conditions were met: (1) the average confidence level of the cross-correlation peak was $R>4$; and (2) none of the template cross-correlations yielded an outlier in the template-object relative velocity. The first condition removed 9 sources from the main sample of 37 objects while the second condition removed five more sources. Figure 1 shows that the rejected sources are mostly close to the faint magnitude limit of our survey. We note that the two brightest sources with unreliable measurements (see also Fig. 1) are those that had been selected as UCD candidates based only on morphology from ACS imaging (ACS Fornax cluster survey, see Jordán et al. 2007). Both sources are located within $2^{\prime}$ to the center of NGC 1399 and are the only objects in our target sample whose coordinates could not be tied to the USNO B2.0 system. We attribute their low flux level to an offset in relative coordinates with respect to the rest of our sample.

A final sample of 23 reliable measurements is obtained, of which 15 have archival HST imaging available. This is the largest homogeneous set of UCDs for which dynamical masses have been derived. The resulting range of intrinsic velocity dispersions is $9<\sigma_{\text {obj }}<36 \mathrm{~km} \mathrm{~s}^{-1}$, with a mean of $24 \mathrm{~km} \mathrm{~s}^{-1}$. These values are listed in Table 1 for the 15 sources with HST imaging, and in Table 3 for the 8 sources without HST imaging. A map and the magnitude distribution of the investigated compact objects is shown in Fig. 1.

\subsection{Mass modelling taking into account aperture effects}

To estimate the masses of the UCDs, we used the mass modelling algorithm outlined in Hilker et al. (2007). This includes a correction of the measured dispersion $\sigma_{\mathrm{obj}}$ to the true global dispersion $\sigma_{\text {obj,cor }}$, due to the fact that our measurements miss the contributions from stars outside the fibre aperture.

The mass modelling involved the following steps:

1. The observed, PSF-deconvolved luminosity profile from HST photometry was parameterized by the best-fitting density law. For most UCDs a satisfactory fit was achieved with a King or generalized King profile. Only UCD3 (ID FLAMES $=$ $F-19)$ required a two component King+Sersic function to be fitted well (see also Evstigneeva et al. 2007). The profile parameters for all 15 sources are shown in Table 2.

2. The 2-dimensional surface density profile was deprojected by means of Abel's integral equation into a 3-dimensional density profile.

3. The cumulated mass function $M(<r)$, the potential energy $\phi(r)$ and the energy distribution function $f(E)$ were calculated from the 3-dimensional density profile.

4. Finally, an $N$-body representation of the UCD was created by using the deprojected density profile and the energy distribution function. For every model, 100000 test particles were distributed and their $x, y$ and $z$ positions and corresponding $v_{x}, v_{y}$ and $v_{z}$ velocities were given as output.

After generating the UCD model, the velocity dispersion as seen by an observer was simulated. In doing so, the following steps were performed:

1. All test particles are convolved with a Gaussian whose fullwidth at half-maximum (FWHM) corresponds to the observed seeing.

2. The fraction of the "light" (Gaussian) that falls into the fibre aperture (1".2 for FLAMES) at the projected distance of the observed object $(19 \mathrm{Mpc})$ is calculated.

3. These fractions are used as weighting factors for the velocities. All weighted velocities that fall into the fibre region are then used to calculate the "mimicked" observed velocity dispersion $\sigma_{\text {mod }}$.

Iteratively, the total "true" mass of the modelled object, $M_{\text {true }}$, that corresponds to the observed velocity dispersion, $\sigma_{\mathrm{obs}}$ is then 

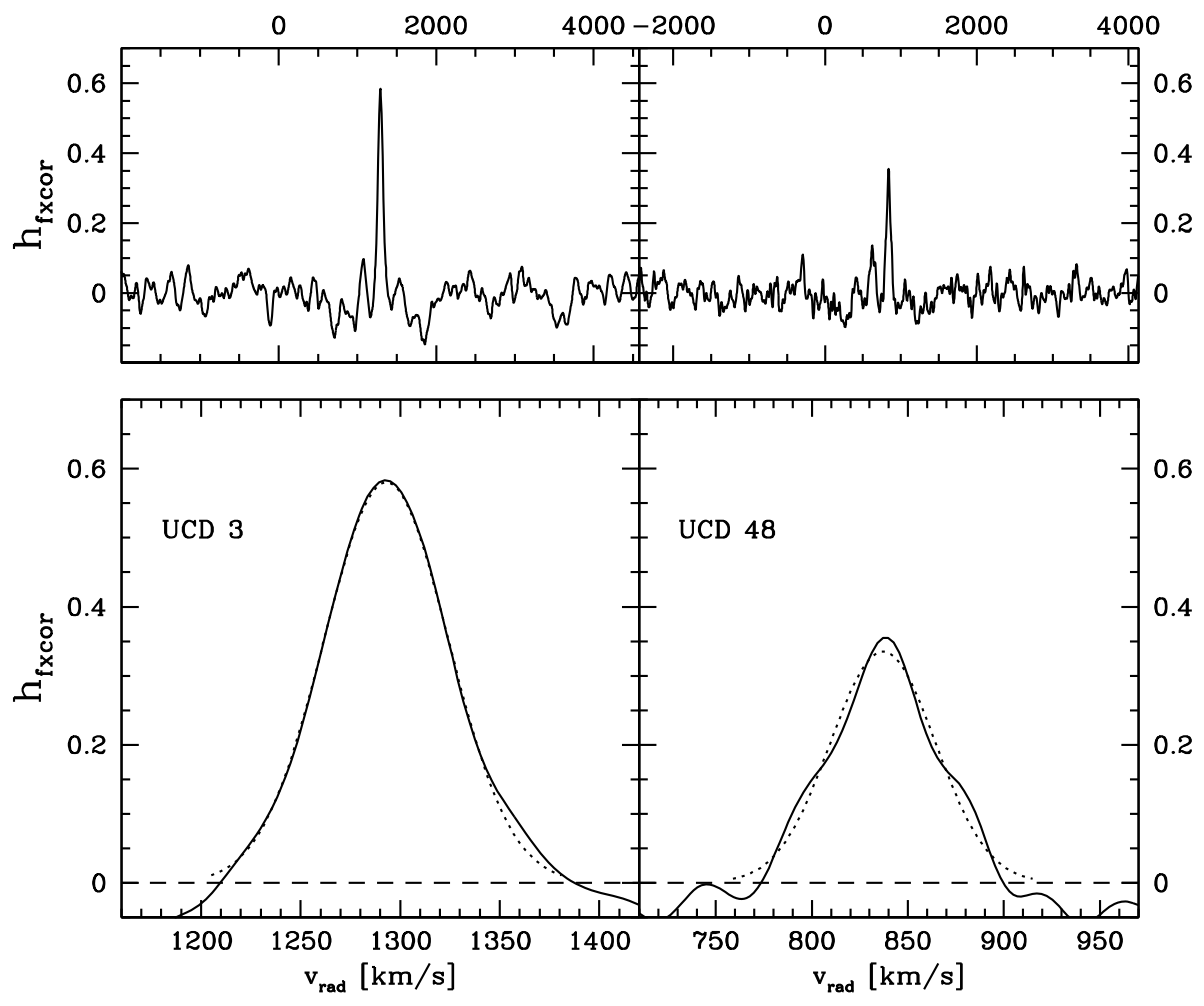

Fig. 4. Plot showing the Fourier crosscorrelation peaks for objects F-19 (UCD3, left) and $\mathrm{F}-22$ (UCD48, right), whose spectra are shown in Fig. 2. The lower panels are magnified views of the overall cross-correlation results from the upper panels. In the lower panels, the Gaussian fit to the cross-correlation peak is indicated by a dotted line. See text for more details.

determined by scaling a first "guess" mass, $M_{\text {guess }}$, via the formula $M_{\text {true }}=M_{\text {guess }} \cdot\left(\sigma_{\text {obs }} / \sigma_{\text {mod }}\right)^{2}$. The masses, mass-to-light ratios, global and central velocity dispersions and the main model parameters derived in this way are listed in Table 1 . Note that the three brightest UCDs in our study were also observed by Hilker et al. (2007) with UVES. The $M / L$ ratios derived for them in that study are indicated in Table 1 . The error ranges of the three estimates overlap, and the average ratio between our $M / L$ values and those from Hilker et al. (2007) is $0.90 \pm 0.16$, consistent with unity.

\section{Results}

With the total mass derived from the dynamical modelling, we calculated the optical mass-to-light ratio $\mathrm{M} / \mathrm{L}_{V}$, using the $V$-band photometry from the wide-field imaging data presented in Hilker et al. (2003) and Mieske et al. (2006, 2007a). Based upon the modelling algorithm of Hilker et al. (2007), we also calculated the central velocity dispersion $\sigma_{0}$ for all sources with HST imaging available. The ratio $\frac{\sigma_{0}}{\sigma}$ was 1.23 on average with a scatter of 0.07 . For those eight sources with reliable $\sigma$ measurements but without HST data (Table 3), we assumed an average correction factor of 1.23 to include them in an analysis of their locus in the $M_{V}-\sigma_{0}$ plane. This plot is shown in Fig. 6.

In the $M_{V}-\sigma_{0}$ plane, the relation for globular clusters and the extrapolation of the Faber-Jackson relation for luminous elliptical galaxies (Faber \& Jackson 1976) intersect at about $M_{V} \simeq-10 \mathrm{mag}$. In the luminosity regime of our sample of Fornax UCDs, both extrapolations bifurcate. We can therefore roughly subdivide our sample into objects closer to the extrapolation of GC relation, and objects closer to the extrapolation of the Faber-Jackson relation (which also happens to match the compact elliptical galaxy M32; Evstigneeva et al. 2007). The three brightest UCDs $\left(M_{V}<-12 \mathrm{mag}\right)$ are clearly more consistent with the Faber-Jackson relation while fainter UCDs are preferentially closer to the GC relation. We find that the

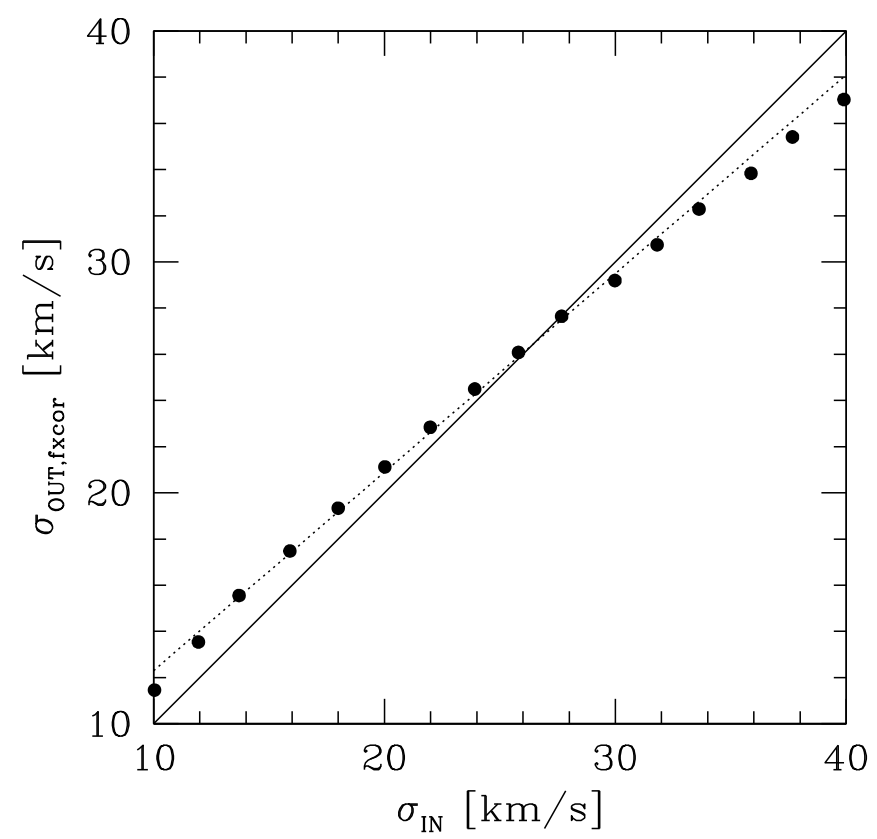

Fig. 5. Plot illustrating the tests performed with cross-correlating artificially broadened spectra. The input width, $\sigma_{\text {IN }}$, is plotted against the width, $\sigma_{\text {OUT }}$, measured using the fxcor task. The solid line shows the identity relation. The dotted line is a linear fit to $\sigma_{\text {OUT }}$ as a function of $\sigma_{\text {IN }}$. The measured values of $\sigma$ are corrected for according to this relation. The values shown here are for the case of $k=3$ as low frequency cut-off. For $k>3$, the deviations from a linear relation were larger.

projected clustercentric distance of objects that are more consistent with the Faber-Jackson relation is $\sim 60 \pm 30 \%$ larger than that of sources more consistent with the GC relation. These findings are consistent with the hypothesis that our sample consists of both objects associated to the globular cluster system of 


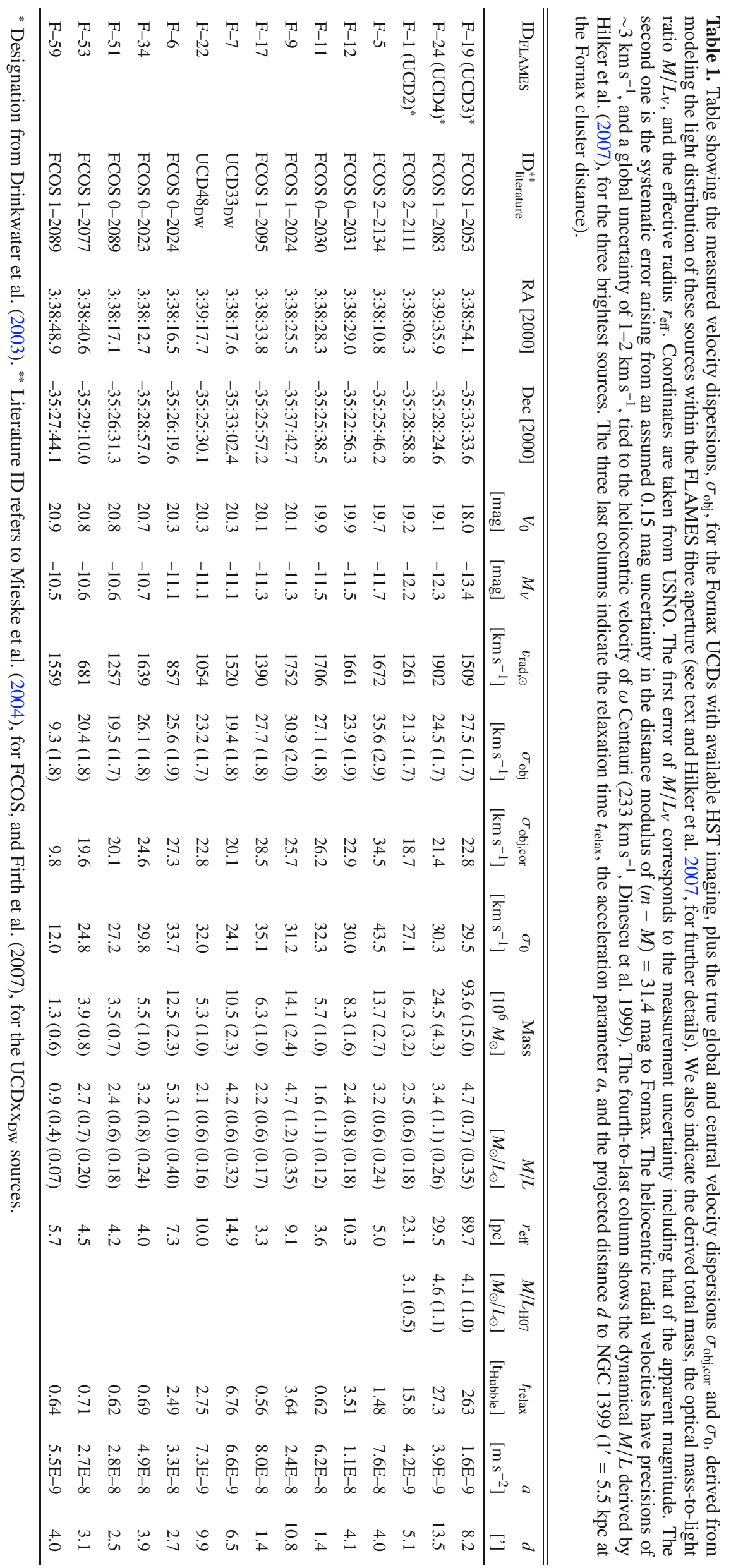


Table 2. Columns 2 to 6 of this table show the structural parameters of the King profile fits for the 15 UCDs from Table 1 (see also Evstigneeva et al. 2008). The King profiles are parametrized as follows: $I(r)=I_{0}\left[\frac{1}{\left(1+\left(r / r_{c}\right)^{2}\right)^{\frac{1}{\alpha}}}-\frac{1}{\left(1+\left(r_{t} / r_{c}\right)^{2}\right)^{\frac{1}{\alpha}}}\right]^{\alpha}$. For the brightest source (F-19), a composite King+Sersic profile was necessary to provide a satisfactory fit. The Sersic profile is parametrized as follows: $I(r)=I_{\text {eff }} \exp \left[-k\left(\left(\frac{r}{r_{\text {eff }}}\right)^{\frac{1}{n}}-1\right)\right]$. The parameters of the Sersic profile are indicated in Cols. 7 to 9.

\begin{tabular}{|c|c|c|c|c|c|c|c|c|}
\hline ID FLAMES & $\mu_{0}\left[\mathrm{mag} / \operatorname{arcsec}^{2}\right]$ & $r_{\mathrm{c}}[\mathrm{pc}]$ & $r_{\mathrm{t}}[\mathrm{pc}]$ & $c$ & $\alpha$ & $\mu_{\mathrm{eff}}\left[\mathrm{mag} / \operatorname{arcsec}^{2}\right]$ & $r_{\mathrm{eff}}[\mathrm{pc}]$ & $n$ \\
\hline $\mathrm{F}-19$ & 16.03 & 4.90 & 230.6 & 1.67 & 2 & 21.34 & 118.9 & 1 \\
\hline $\mathrm{F}-24$ & 15.11 & 3.03 & 4501.2 & 3.17 & 3.32 & - & - & - \\
\hline $\mathrm{F}-1$ & 14.81 & 2.23 & 487.1 & 2.34 & 1.23 & - & - & - \\
\hline $\mathrm{F}-5$ & - & 1.22 & 77.1 & 1.80 & 2 & - & - & - \\
\hline $\mathrm{F}-12$ & 13.66 & 1.24 & 72.9 & 1.77 & 1.25 & - & - & - \\
\hline $\mathrm{F}-11$ & - & 1.70 & 0.98 & 49.1 & 2 & - & - & - \\
\hline F-9 & - & 1.48 & 3.14 & 95.0 & 2 & - & - & - \\
\hline F-17 & - & 1.70 & 0.90 & 45.0 & 2 & - & - & - \\
\hline $\mathrm{F}-7$ & 16.23 & 7.03 & 96.3 & 1.14 & 2.79 & - & - & - \\
\hline $\mathrm{F}-22$ & 11.67 & 0.39 & 102.8 & 2.52 & 1.20 & - & - & - \\
\hline F-6 & - & 1.57 & 2.29 & 85.0 & 2 & - & - & - \\
\hline F-34 & - & 1.51 & 1.30 & 42.2 & 2 & - & - & - \\
\hline F-51 & - & 2.45 & 0.49 & 138.8 & 2 & - & - & - \\
\hline F-53 & - & 1.95 & 0.91 & 81.5 & 2 & - & - & - \\
\hline F-59 & - & 1.48 & 1.97 & 59.5 & 2 & - & - & - \\
\hline
\end{tabular}

Table 3. Measured velocity dispersions $\sigma_{\mathrm{obj}}$ for the Fornax UCDs without available HST imaging. IDs are from literature sources as in Table 1.

\begin{tabular}{llrrrrrr}
\hline \hline ID $_{\text {FLAMES }}$ & ID $_{\text {literature }}^{*}$ & RA [2000] & Dec [2000] & $V_{0}[\mathrm{mag}]$ & $M_{V}[\mathrm{mag}]$ & $v_{\text {rad,॰ }}\left[\mathrm{km} \mathrm{s}^{-1}\right]$ & $\sigma_{\text {obj }}\left[\mathrm{km} \mathrm{s}^{-1}\right]$ \\
\hline F-3 & UCD27 $_{\text {DW }}$ & $3: 38: 10.4$ & $-35: 24: 06.2$ & 19.7 & -11.7 & 1626 & $31.3(1.5)$ \\
F-18 & UCD44 & $3: 38: 42.0$ & $-35: 33: 13.0$ & 19.7 & -11.7 & 2024 & $19.1(1.4)$ \\
F-23 & UCD49 & $3: 39: 20.5$ & $-35: 19: 14.2$ & 19.7 & -11.7 & 1480 & $21.9(1.4)$ \\
F-2 & FCOS 2-2153 & $3: 38: 06.5$ & $-35: 23: 04.0$ & 20.0 & -11.4 & 1426 & $18.7(1.4)$ \\
F-8 & FCOS 0-2066 & $3: 38: 23.2$ & $-35: 20: 00.7$ & 20.1 & -11.3 & 1414 & $25.7(1.4)$ \\
F-40 & 92.099* & $3: 37: 52.5$ & $-35: 28: 57.9$ & 20.7 & -10.7 & 1497 & $27.3(1.4)$ \\
F-60 & FCOS 2-2100 & $3: 38: 00.2$ & $-35: 30: 08.2$ & 20.9 & -10.5 & 871 & $24.3(1.9)$ \\
F-64 & FCOS 1-2080 & $3: 38: 41.4$ & $-35: 28: 46.6$ & 21.0 & -10.4 & 1728 & $24.7(1.7)$ \\
\hline
\end{tabular}

* This source designation is from Richtler et al. (2008).

NGC 1399, and objects with more complex dynamical formation history, being associated more to the overall cluster potential.

In Fig. 7 we plot metallicity $\mathrm{Z} / \mathrm{H}$ against $M / L$ ratio. The metallicity $\mathrm{Z} / \mathrm{H}$ is derived directly for some sources from previous spectroscopy (Mieske et al. 2006), for others derived from their $(V-I)$ colour (Mieske et al. 2007a), using the calibration of Kissler-Patig et al. (1998). This calibration was shown to be accurate to within 0.1-0.2 dex (Mieske et al. 2006) for old stellar populations with $[\mathrm{Fe} / \mathrm{H}] \gtrsim-1.0$ dex. In the plot we indicate SSP predictions from Bruzual \& Charlot (2003) and Maraston et al. (2005) for $M / L$ ratios of populations with solar $[\alpha / \mathrm{Fe}]$ abundances, with ages between 5 and 13 Gyr. The former models assume a Chabrier IMF (Chabrier 2003), while the latter models assume a Kroupa IMF (Kroupa 2001). We note that both IMFs are very similar and do not account for the difference in predicted $M / L$ ratios at fixed age and metallicity (see also Dabringhausen et al. 2008). Rather, it is the choice of different stellar evolutionary codes which leads to the $20 \%$ differences between Bruzual $\&$ Charlot and Maraston $M / L$ predictions. Most of the $M / L$ data points are consistent to within their errors with the theoretical predictions assuming a canonical IMF, with three sources showing somewhat elevated $\mathrm{M} / \mathrm{Ls}$.

In the two top panels of Fig. 8 we plot $M_{V}$ and mass vs. the $M / L$ ratio for the 15 sources with HST imaging. We indicate the faint magnitude limit of our survey, which translates into a mass dependent $M / L$ sensitivity limit. To test whether the rise of $M / L$ with mass generally observed in the regime $10^{5}<M / M_{\odot}<10^{8}$ can be traced by our data, we fit a linear relation to the distribution of mass vs. $M / L$ ratio. We find a slope different from 0 at the $2.8 \sigma$ level. The significance of the slope was calculated by random resampling of the data points around the fitted relation. For this re-sampling, the scatter of the data points around the relation was used, given that it was about $25 \%$ larger than the average error of the data points.

There are two caveats regarding the interpretation of this $\sim 3 \sigma$ slope. The first caveat is the mass dependent $M / L$ sensitivity limit. Assuming a random distribution of $M / L$ ratio with $M_{V}$, a fixed magnitude limit will artificially create a slope in the mass $-M / L$ plane, due to the lack of sources at low masses and high $M / L$ ratio. The second caveat is that a relation between mass and $M / L$ is naturally produced if there is a relation between mass and metallicity (see e.g. Mieske et al. 2004 and 2006). This is because optical $M / L$ ratios increase towards higher metallicites (see Fig. 7). We correct for this effect by normalising our $M / L$ ratios to solar metallicity (see also Mieske \& Kroupa 2008, and Dabringhausen et al. 2008). To this end, we fit a relation

$M / L_{\text {theo }}=a+b \times \exp (c \times[\mathrm{Fe} / \mathrm{H}])$

to the $M / L$ predictions for a $13 \mathrm{Gyr}$ population from each of the two model sets of Bruzual \& Charlot (2003) and Maraston (2005) (see Fig. 7). We define the mean of the two fits as the reference relation: $M / L_{\text {theo }}=0.5 \times\left(M / L_{\text {theo, } \mathrm{BC} 03}+M / L_{\text {theo, } \mathrm{M} 05}\right)$. 


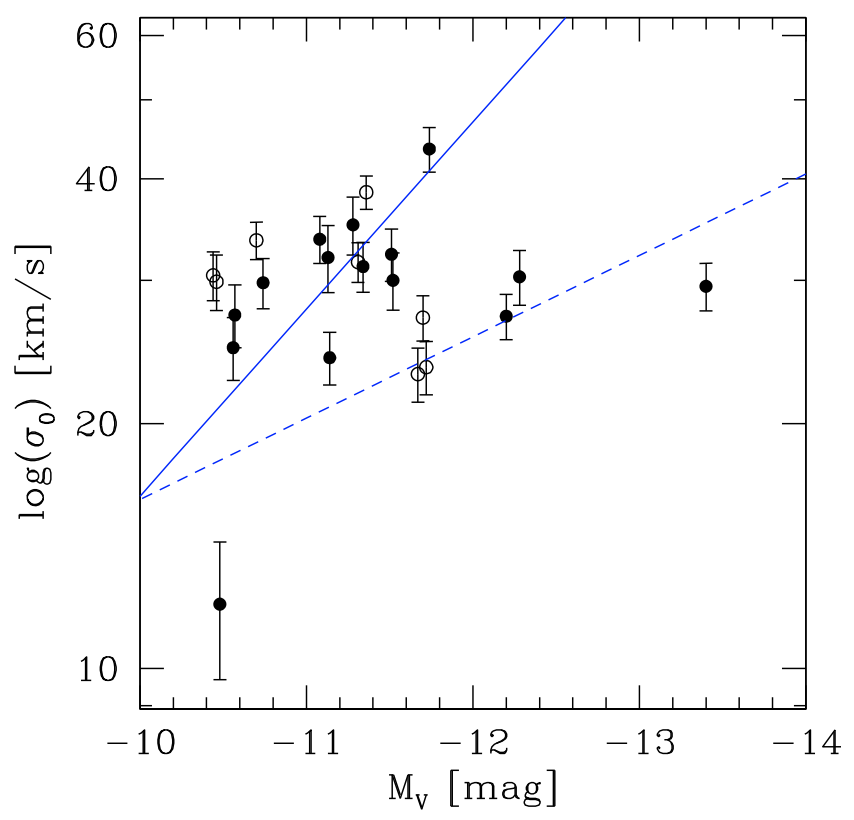

Fig. 6. Absolute magnitude of UCDs plotted against their central velocity dispersion $\sigma_{0}$. The solid line is the extrapolation of the relation defined by Galactic GCs (McLaughlin \& van der Marel 2005), the dashed line is the extrapolation of the Faber-Jackson relation for luminous elliptical galaxies (Faber \& Jackson 1976), which also fits the compact elliptical M32. Open symbols indicate objects without HST imaging, for which the average correction factor between global $\sigma$ and $\sigma_{0}$ was assumed as derived from modelling of the 15 objects with HST imaging.

See Mieske \& Kroupa (2008) for the details of these fits. We then normalise our $M / L$ values in the following way:

$(M / L)_{\text {normalised }}=\frac{(M / L)}{(M / L)_{\text {theo }}} *(M / L)_{\text {theo }, 0}$

$(M / L)_{\text {theo, }, 0}$ is the theoretical prediction for $[\mathrm{Fe} / \mathrm{H}]=0$.

The normalised values are plotted vs. mass in the bottom left panel of Fig. 8. Their errors include the difference between the model predictions and an assumed uncertainty of 0.3 dex in $[\mathrm{Fe} / \mathrm{H}]$, although note that these contributions are small compared to the uncertainty of the $M / L$ measurement itself. The significance of the correlation decreases to $1.5 \sigma$ (and $1.2 \sigma$ when excluding the lowest mass data point). Our data are hence consistent with a non-correlation between mass and $M / L$ ratio in the mass range $3 \times 10^{6}<M<10^{8} M_{\odot}$, especially when taking into account the mass dependent selection limit. We find no extremely high M/L ratios as in Virgo (Haşegan et al. 2005).

In the bottom right panel of Fig. 8 we plot the normalised $M / L$ ratio vs. half-mass relaxation time $t_{\text {relax }}$ (see also Table 1 ). The relaxation time is a more direct measure of the state of dynamical evolution than the mass. We use the following equation from Dabringhausen et al. (2008, Eq. (6) in their paper) to calculate $t_{\text {relax }}$ in units of Myrs:

$t_{\text {relax }}=\frac{0.234}{\log (M)} * \sqrt{M * r_{\mathrm{eff}}^{3} / 0.0045}$.

This formula is based on Spitzer \& Hart (1971) and Spitzer (1987). The mass $M$ is given in solar masses and $r_{\text {eff }}$ in pc. We find only a very marginal trend $(2.1 \sigma)$ of increasing $M / L$ ratio with increasing $t_{\text {relax }}$. A larger sample over a broader mass range is required to quantify a trend of $M / L$ with mass or relaxation time (Sect. 5.1).

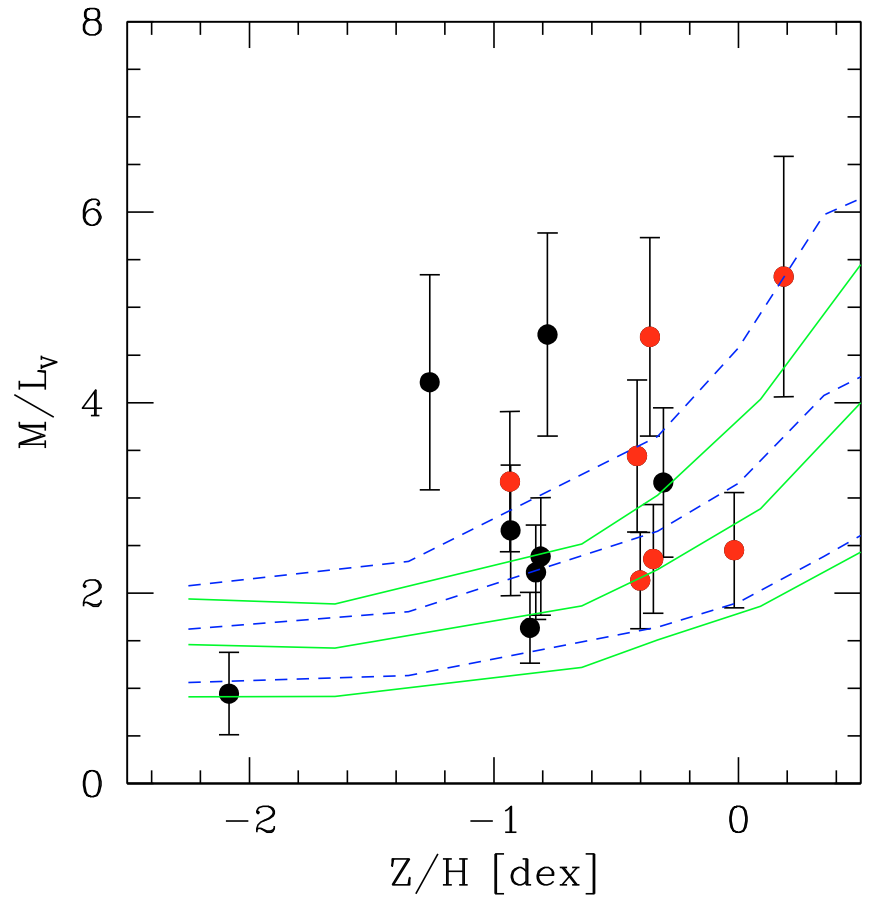

Fig. 7. Z/H of the UCDs plotted against their $M / L$ ratios. Solid (green) lines indicate stellar population models from Bruzual \& Charlot (2003) for ages (from bottom to top) of 5, 9, and $13 \mathrm{Gyr}$, assuming a Chabrier IMF. Blue (dashed) curves are from Maraston et al. (2005) for the same age ranges, assuming a Kroupa IMF. The $\mathrm{Z} / \mathrm{H}$ values are from Mieske et al. (2006), assuming a solar $[\alpha / \mathrm{Fe}]$ abundance, as suggested in Mieske et al. (2007b). Red data points indicate sources for which spectroscopic $[\mathrm{Fe} / \mathrm{H}]$ estimates are available. For the remaining sources, $[\mathrm{Fe} / \mathrm{H}]$ is estimated from their $(V-I)$ colour, using the transformation of Kissler-Patig et al. (1998) (see also Mieske et al. 2006).

In Fig. 9 we plot $M / L$ ratio vs. the acceleration parameter $\mathrm{a}=\frac{\mathrm{G} \times \mathrm{M}}{\mathrm{r}_{\mathrm{h}}^{2}}$ to illustrate that the internal dynamics of the compact objects are far from the MOND (Milgrom 1983) regime of weak acceleration $\left(\mathrm{a}_{0} \sim 1.2^{-10} \mathrm{~m} \mathrm{~s}^{-2}\right)$. Figure 9 also shows the $M / L$ ratio as a function of projected distance d to NGC 1399. Table 1 lists both the acceleration parameter a and the projected distance $d$. In the case that tidal heating efficiently increases the internal velocity dispersion of sources with small apocentric radii (Fellhauer \& Kroupa 2006), one may expect a trend of increasing $M / L$ with decreasing radius. No such trend is seen.

\section{Discussion}

\section{1. $M / L$ ratio measurements over a range of environments}

In order to further quantify the dependence of $M / L$ ratio on mass, and to investigate $M / L$ variation with environment, we combine our data for Fornax UCDs with $M / L$ ratio measurements of other compact stellar systems from literature studies, see Fig. 12. This covers the regime of low-mass Milky Way globular clusters $\left(M \gtrsim 5 \times 10^{4} M_{\odot}\right)$ up to the most massive UCDs $\left(M \sim 10^{8} M_{\odot}\right)$.

\subsubsection{Revision of literature mass estimates}

In Fig. 12 we use revised dynamical mass estimates of the Cen A globular clusters from Rejkuba et al. (2007) and the DGTOs from Haşegan et al. (2005). Those estimates are obtained by 
applying the same mass modelling and aperture simulations as presented in Sect. 3.3 and in Hilker et al. (2007). To this end, we assumed King models as representations of the light profiles with the projected half-light radii and King concentrations as given in the two papers. We include new measurements from five additional Cen A globular clusters (Rejkuba et al. 2007), using structural parameters provided by Gomez from observations with IMACS on Magellan (private communication).

It turned out that the modelled masses (and thus mass-tolight ratios) of the Cen A globular clusters are up to $30 \%$ lower than estimated from the virial mass estimator, whereas most of the modelled DGTO masses are in general higher (up to $60 \%$ ). Figure 10 compares our modelled masses with the dynamical estimates given in the literature. The reason for the discrepancies is the different treatment of the aperture corrections that have to be applied to the observed velocity dispersions.

In case of the Cen A data the authors estimated the aperture corrections to be a few percent, but they preferred to assign these corrections only to the total error budget, and instead used directly the observed $\left(\sigma_{\text {obs }}\right)$ values with the virial estimator to derive masses. However, at the distance of Cen A, 3.84 Mpc (Rejkuba 2004), the ratio between the projected half-light diameter (4-48 pc) and the slit width $\left(1^{\prime \prime} \simeq 19 \mathrm{pc}\right)$ is such that aperture corrections can not be neglected. A large fraction of the light of the most extended massive clusters lies outside the slit area which prohibits the measurement of a global velocity dispersion. The ratios $\sigma_{\text {tot }} / \sigma_{\mathrm{obs}}$ and $\sigma_{0} / \sigma_{\mathrm{obs}}$ as function of projected half-light radius are shown in Fig. 11.

In case of the DGTO data, the authors derived the dynamical mass from the King mass estimator (e.g., Dubath \& Grillmair 1997), for which the core radius and the central velocity dispersion have to be known. Haşegan et al. (2005) corrected the measured velocity dispersion to the central one by "scaling upward to account for the blurring of the actual velocity dispersion profiles within the ESI slit" for which the knowledge of the intrinsic light profile from HST imaging has to be known. The Virgo cluster is far enough away, 16.1 Mpc (Tonry et al. 2001), that most of the light of the DGTOs, which have half-light diameter between 7 and $58 \mathrm{pc}$, falls into the slit (with width of $0.75 \simeq 58 \mathrm{pc}$ ). One tends to measure the global velocity dispersion for most of the DGTOs. Thus, the correction to central velocity dispersions should be larger for smaller objects, since for those the measured dispersion is closest to the global one. This, however, is opposite to the trend of the corrections applied by Haşegan et al. (2005) (see their Tables 4 and 5), such that on average the masses derived by Haşegan et al. (2005) have to be corrected upwards (see Fig. 10). We will take our modelling results for the further discussions. The revised model masses will also be included in an upcoming paper (Haşegan et al. 2008, in preparation), along with $M / L$ measurements of newly discovered Virgo DGTOs.

\subsubsection{A trend of $M / L$ with mass and relaxation time}

In Fig. 12, we plot both, mass and relaxation time, vs. direct and normalised $M / L$ ratios for our compiled sample of compact stellar systems. In Table 2 , the $M / L$ ratios, metallicities and sizes for these objects are shown. When fitting a linear relation to relaxation time as a function of mass, we find that relaxation time equal to 1 Hubble time is reached at a mass of about $2 \times 10^{6} M_{\odot}$. In the following we adopt this mass as an approximate limit between globular clusters and UCDs (see also Mieske \& Kroupa 2008).
Figure 12 shows a clear rise of the $M / L$ ratio for masses above $\simeq 2 \times 10^{6} M_{\odot}$. When correcting the $\mathrm{M} / \mathrm{L}$ ratio measurements for their metallicity dependence, this is still clearly visible (see also Mieske \& Kroupa 2008; Dabringhausen et al. 2008), formally significant at the $8 \sigma$ limit. The $\mathrm{M} / \mathrm{L}$ ratio distribution of objects below and above the $2 \times 10^{6} M_{\odot}$ limit stems from the same parent distribution with only the $2.7 \times 10^{-11}$ probability, according to a KS test. The average normalised $M / L$ ratio for globular clusters is $2.70 \pm 0.17$, while it is $5.44 \pm 0.37$ for UCDs. The rise in $M / L$ corresponds to a $40 \%$ increase of normalised $M / L$ ratio per mass decade: $\frac{\operatorname{dlog}(M / L))}{\operatorname{dlog}(M)}=0.147 \pm 0.019$ (applying a $3 \sigma$ clipping). A separation between UCDs and GCs at $t_{\text {relax }}=1 t_{\text {Hubble leaves the mean } M / L \text { ratios of GCs and UCDs }}$ unchanged with respect to the mass cut at $2 \times 10^{6} M_{\odot}$. For the separation at $t_{\text {relax }}=1 t_{\text {Hubble }}$, the $M / L$ distributions have a common parent distribution at a probability of $1.4 \times 10^{-10} . M / L$ scales with relaxation time almost in the same way as with mass: $\frac{\operatorname{dlog}(M / L))}{\operatorname{dlog}\left(t_{\text {relax }}\right)}=0.150 \pm 0.021$.

Apart from studying the relative difference in $M / L$ ratio between GCs and UCDs, it is also important to compare the $M / L$ ratios with the model predictions on an absolute scale. While the average $M / L$ of UCDs is $\sim 40 \%$ above the 13 Gyr isochrone, $M / L$ ratios of the Galactic globular clusters are below the $13 \mathrm{Gyr}$ isochrone by the same factor. The mean age derived from their location with respect to the isochrones is 7-8 Gyr, well below the typical globular cluster age of $\sim 12-13 \mathrm{Gyr}$, indicating that the input for the stellar population codes may not represent the globular cluster properties (see also Dabringhausen et al. 2008). In what follows, we therefore discuss the extent to which dynamical evolution may have changed $M / L$ ratios for the compact stellar systems under investigation, and hence contributed to shaping the observed trend between mass and M/L.

\subsubsection{Does dynamical evolution shape the $M / L$ trend?}

Baumgardt \& Makino (2003) showed that star clusters experience a depletion in low-mass stars leading to a drop in $\mathrm{M} / \mathrm{L}$ of up to 0.5 after about 0.8 dissolution timescales. This drop in $\mathrm{M} / \mathrm{L}$ corresponds to about $30 \%$ of the mean measured $M / L$ ratio of Galactic globular clusters. Dynamical evolution could hence be responsible for the lower $M / L$ ratios of GCs, provided that their dissolution timescale is comparable to or smaller than a Hubble time. To estimate the dissolution timescale $t_{\text {diss }}$ for a typical Galactic globular cluster, we apply Eq. (6) from the recent study of Lamers et al. (2006), in which the photometric evolution of dissolving star clusters in the Galaxy's gravitational potential is investigated.

$t_{\text {diss }}=6.60 \times 10^{2}\left(\frac{M_{i}}{10^{4}}\right)^{0.653} \times t_{0}^{0.967-0.00825 \times \log \left(M_{\mathrm{i}} / 10^{4}\right)}$.

$M_{i}$ is the initial cluster mass. The time-scale $t_{0}$ depends on the tidal field of the environment. Lamers et al. (2006) adopt $t_{0}=21.8 \mathrm{Myr}$, which is valid for a circular orbit in the Galaxy at $8.5 \mathrm{kpc}$ radial distance (Baumgardt \& Makino 2003). The median galactocentric distance of the Galactic GCs plotted in Fig. 12 is 9.2 kpc (McLaughlin \& van der Marel 2005), very close to the assumed $8.5 \mathrm{kpc}$. Therefore, we also adopt $t_{0}=$ $21.8 \mathrm{Myr}$ for estimating $t_{\text {diss }}$. Evaluating Eq. (4), we obtain $t_{\text {diss }} \simeq$ $50 \mathrm{Gyrs}$ for $10^{5} M_{\odot}$, and $t_{\text {diss }} \simeq 250 \mathrm{Gyrs}$ for $10^{6} M_{\odot}$. That is, dynamical evolution should not have changed the primordial $M / L$ ratios of compact stellar systems with masses above $10^{6} M_{\odot}$, including the UCDs. For Galactic GCs in Fig. 12, masses are between $10^{4}$ and $10^{6} M_{\odot}$, such that their dissolution timescales 

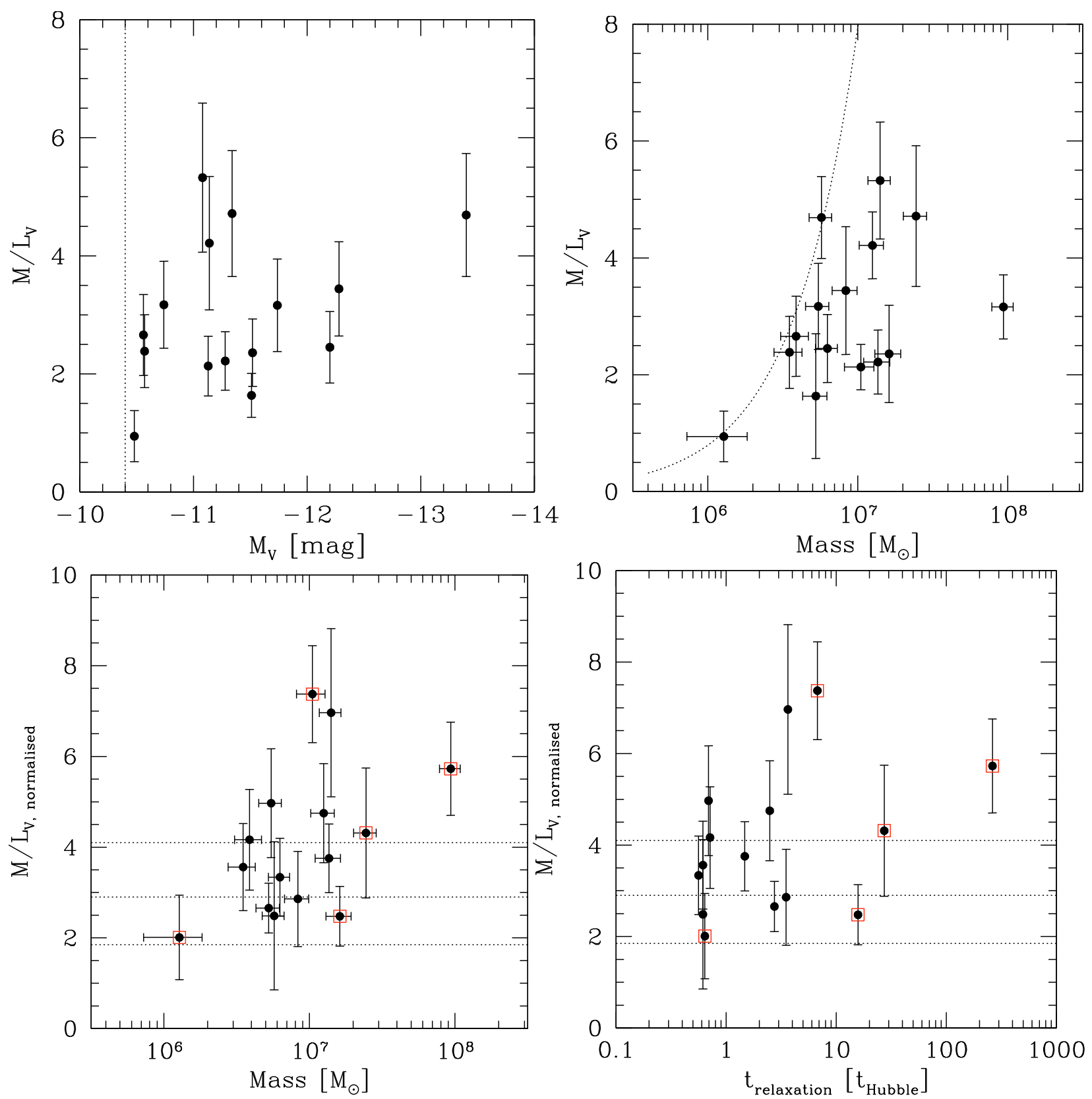

Fig. 8. Top left: absolute magnitude of UCDs plotted against their $M / L$ ratio in the $V$-band. The dotted line indicates the faint magnitude limit of our survey. Top right: Masses of the UCDs from the left plot, plotted against their $M / L$ ratio. The dotted line indicates the mass dependent upper limit of our $M / L$ sensitivity, caused by our faint magnitude limit of $M_{V}=-10.4$ mag. There is a $3 \sigma$ correlation between the shown data points. Bottom left: plot analogous to the top right panel. Now, the $M / L$ ratio measurements have been normalised to the same (solar) metallicity (see text). For this, we assume the mean of the model predictions from Bruzual \& Charlot (2003) and Maraston (2005) in Fig. 7. The horizontal lines indicate the $M / L$ ratios from the model predictions for 13,9 , and $5 \mathrm{Gyr}$ (top to bottom). There is only a $1.5 \sigma$ correlation between the data points. Sources marked by large squares are those closer to the Faber Jackson relation in Fig. 6. Bottom right: as in the plot on the left, but now the $x$-axis is relaxation time.

are closer to, and in some cases below, a Hubble time. Assuming that the $\mathrm{GC} \mathrm{M} / \mathrm{L}$ ratio decreases linearly up to a difference of 0.5 after 0.8 dissolution timescales (Baumgardt \& Makino 2003), we plot in the bottom left panel of Fig. 12 the expected $M / L$ ratios of Galactic GCs if there was no dynamical evolution. The corrections are small - on average about $5 \%$, which is neglibile in the context of this discussion. Provided that the absolute scale of dissolution times derived by Baumgardt \& Makino (2003) is applicable to the Milky Way GCs included in this study, their $M / L$ ratios should not have notably decreased due to dynamical effects from their initial value.
The stellar population models used here indeed appear to over-estimate the $M / L$ ratios of globular clusters with a canonical IMF by $\sim 40 \%$, and on average under-estimate the $M / L$ ratios of UCDs by about the same amount.

\subsection{Environmental dependence of $M / L$ ratios}

From Fig. 12 it is evident that $M / L$ ratio measurements of the Fornax UCDs fit well into the general trend of $M / L$ increasing with mass. However, it is also interesting to note that we have 

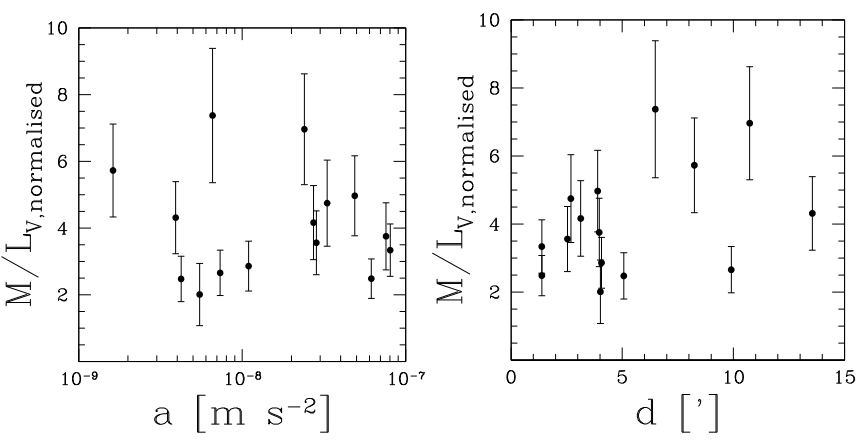

Fig. 9. Left panel: gravitational acceleration, $a=\frac{G \times M}{r_{h}^{2}}$, plotted against normalised $M / L$ ratio. None of the objects are in the low acceleration regime $a \lesssim 1.2 \times 10^{-10} \mathrm{~m} \mathrm{~s}^{-2}$ where MOND has been postulated to hold. Right panel: projected distance to NGC 1399 plotted against normalised $M / L$ ratio.

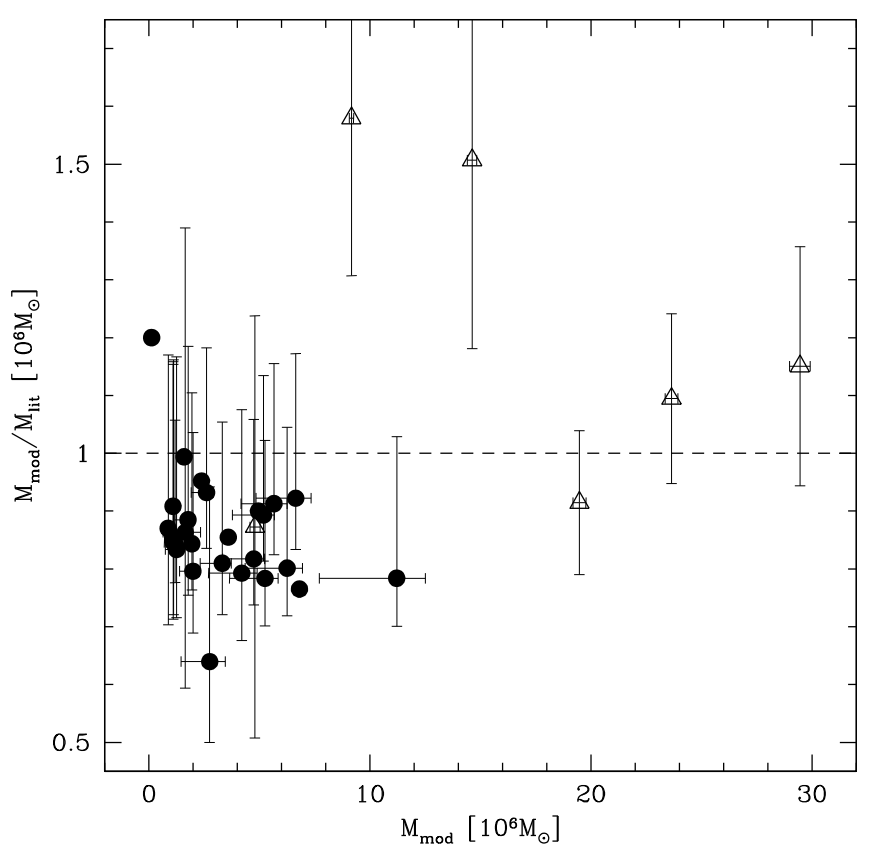

Fig. 10. The effect of proper aperture corrections on dynamical mass estimates. The ratio between modelled mass and dynamical mass as given in the literature is plotted vs. modelled mass. The masses of Cen A GCs from Rejkuba et al. (2007) (solid circles) were previously overestimated by up to $30 \%$, whereas the masses of DGTOs in Virgo from Haşegan et al. (2005) (open triangles) were mostly underestimated. The errorbars reflect the uncertainties given for the literature values.

not found a Fornax UCD with such extraordinarily high $M / L$ ratios as the three DGTOs from Haşegan et al. (2005). Fornax UCDs cover the same mass range as Virgo UCDs, but their average $M / L$ ratio is only $0.61 \pm 0.11$ that of the Virgo UCDs, or $0.71 \pm 0.08$ when excluding the Virgo UCD with the highest $M / L$ ratio (S999).

Such a $M / L$ ratio difference may arise from age differences. If Fornax UCDs have luminosity weighted ages around 7 Gyrs and Virgo UCDs ages around a Hubble time, both populations would be equally inconsistent with $M / L$ ratio predictions from stellar populations for their age, indicating the presence of (baryonic or non-baryonic) dark matter (Mieske \& Kroupa 2008; Dabringhausen et al. 2008). Derivation of spectroscopic ages from line abundances for Virgo UCDs have shown that

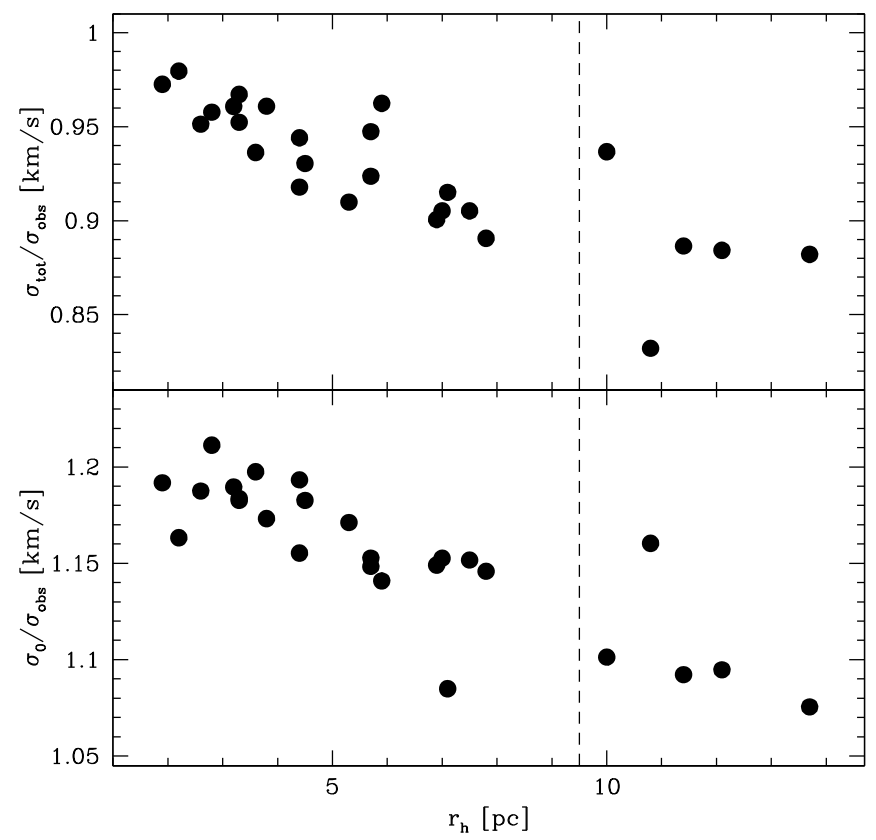

Fig. 11. Corrections from observed to total and central velocity dispersions for Cen A GCs based on our mass modelling. The upper panel shows the ratio between total and observed velocity dispersion, while the lower panel shows the ratio between central and observed velocity dispersion. The dashed vertical line indicates half the width of the slit aperture.

they are most consistent with old ages around a Hubble time (Evstigneeva et al. 2007). For Fornax, the situation is less clear. Mieske et al. (2006) find indications for intermediate ages in Fornax UCDs from relating $\mathrm{H}_{\beta}$ to metallicity sensitive line indices, but these data were not calibrated to the Lick system. Accurate age determinations for a comprehensive sample of Fornax UCDs by spectroscopy or multi-band photometry will allow to draw firmer conclusions in this context.

The lower $M / L$ ratios of Fornax UCDs - provided they are not explained by age differences - may support the assumption of different competing formation channels for UCDs (Mieske et al. 2006). In that context, the higher $M / L$ ratios of Virgo UCDs could be interpreted as being due to dark matter. Goerdt et al. (2008) show that under certain conditions, remnants of tidally stripped dwarf galaxies can maintain a significant amount of dark matter (although see Bekki et al. 2003, for a different view). The lower $M / L$ ratios in Fornax UCDs could then be explained by them being stellar super clusters formed without dark matter (Fellhauer \& Kroupa 2002; Mieske et al. 2006).

\subsection{Fundamental scaling relations of stellar systems: how do UCDs and GCs fit in?}

Here we analyse how compact stellar systems such as UCDs and GCs fit into fundamental scaling relations for more extended stellar systems. In motivating this analysis, we show in Fig. 13 the half-light radius of the compact stellar systems from Fig. 12 plotted against their $M / L$ ratio. We mark the radius at which the relaxation time is equal to one Hubble time, which is roughly $7 \mathrm{pc}\left(\log \left(r_{\mathrm{h}}\right)=0.82\right.$; or $\left.\log \left(r_{\mathrm{h}} / \mathrm{kpc}\right)=-2.18\right)$. As also seen in Fig. 12, this limit nicely marks the rise of $M / L$ ratios between the regime of GCs and UCDs. 

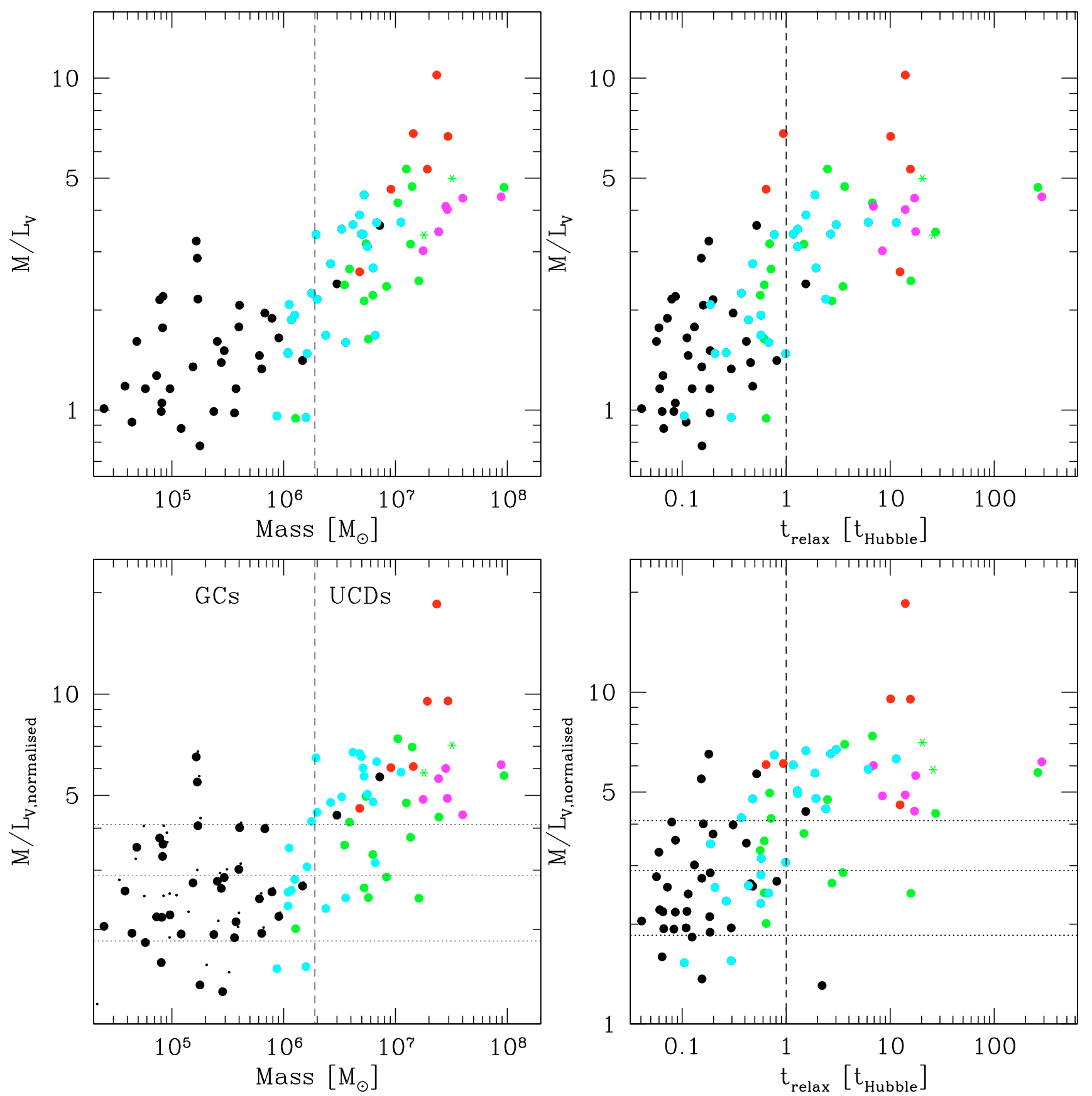

Fig. 12. Top left panel: mass vs. $M / L$ for the isolated compact objects of this paper (green dots) plus literature values of compact objects in CenA (Rejkuba et al. 2007, cyan), Virgo (Haşegan et al. 2005, red; Evstigneeva et al. 2007, magenta), Fornax (Hilker et al. 2007, green asterisks), and Milky Way globular clusters (McLaughlin \& van der Marel 2005, black dots). The vertical dashed line indicates the approximate mass where the relaxation time is equal to one Hubble time (see also the right panels). Top right panel: like the left panel, but plotting relaxation time instead of mass on the $x$-axis. Bottom left panel: like the top left panel, but here all $M / L$ ratio estimates have been normalised to solar metallicity (see text). The horizontal dotted lines indicate the $M / L$ ratios expected for single stellar populations of age 13 , 9 , and 5 Gyrs (from top to bottom) based on Bruzual \& Charlot (2003) and Maraston et al. (2005). The small (black) dots indicate the present-day $M / L$ ratios of the Galactic GCs if they would not have undergone dynamical evolution (see text). Bottom right panel: like the left panel, but plotting relaxation time instead of mass on the $x$-axis.

In Fig. 14 we show the location of all compact stellar systems from Fig. 12 in the so-called "fundamental manifold" (Zaritsky et al. 2006a,b; Zaritsky et al. 2008). The fundamental manifold concept aims at a unifying empirical description of the structural and kinematic properties of stellar spheroids. It relates the effective radius $r_{\mathrm{e}}$ to velocity dispersion $\sigma$ and effective $I$-band surface brightness $I_{\mathrm{e}}$. In their studies, Zaritsky et al. show that stellar spheroids from the scale of galaxy clusters $\left(\sim 10^{5} \mathrm{pc}\right)$ down to the scale of dwarf elliptical galaxies $\left(\sim 10^{2} \mathrm{pc}\right)$ appear to form a common sequence in this manifold. With the data from Fig. 12 we can extend these considerations down to the smallest stellar systems $\left(\sim 10^{0} \mathrm{pc}\right)$.

To derive $I_{\mathrm{e}}$ for the compact stellar systems, we use ( $V-$ $I)$ measurements where available, and otherwise convert $[\mathrm{Fe} / \mathrm{H}]$ 
Table 4. Fitting coefficients for $\log \left(\Upsilon_{\mathrm{e}}^{f}\left(\log (\sigma), \log \left(I_{\mathrm{e}}\right)\right)\right.$ in the three fundamental manifold formulations from Fig. 14. The functional form is $\log \left(\Upsilon_{\mathrm{e}}^{f}\right)=c 1+c 2 * \log (\sigma)+c 3 * \log \left(I_{\mathrm{e}}\right)+c 4 *\left(\log \left(I_{\mathrm{e}}\right)\right)^{2}+c 5 *(\log (\sigma))^{2}+c 6 * \log (\sigma) * \log \left(I_{\mathrm{e}}\right)$

\begin{tabular}{lrrrrrr}
\hline \hline Manifold formulation & $\mathrm{c} 1$ & $\mathrm{c} 2$ & $\mathrm{c} 3$ & $\mathrm{c} 4$ & $\mathrm{c} 5$ & $\mathrm{c} 6$ \\
\hline 1 & 2.75 & -1.70 & -0.295 & - & 0.63 & - \\
2 & 1.8974 & 0.1896 & -0.9699 & 0.1095 & 0.1193 & 0.02893 \\
3 & 2.2397 & -0.3006 & -0.8726 & 0.1159 & 0.2827 & -0.0337 \\
\hline
\end{tabular}

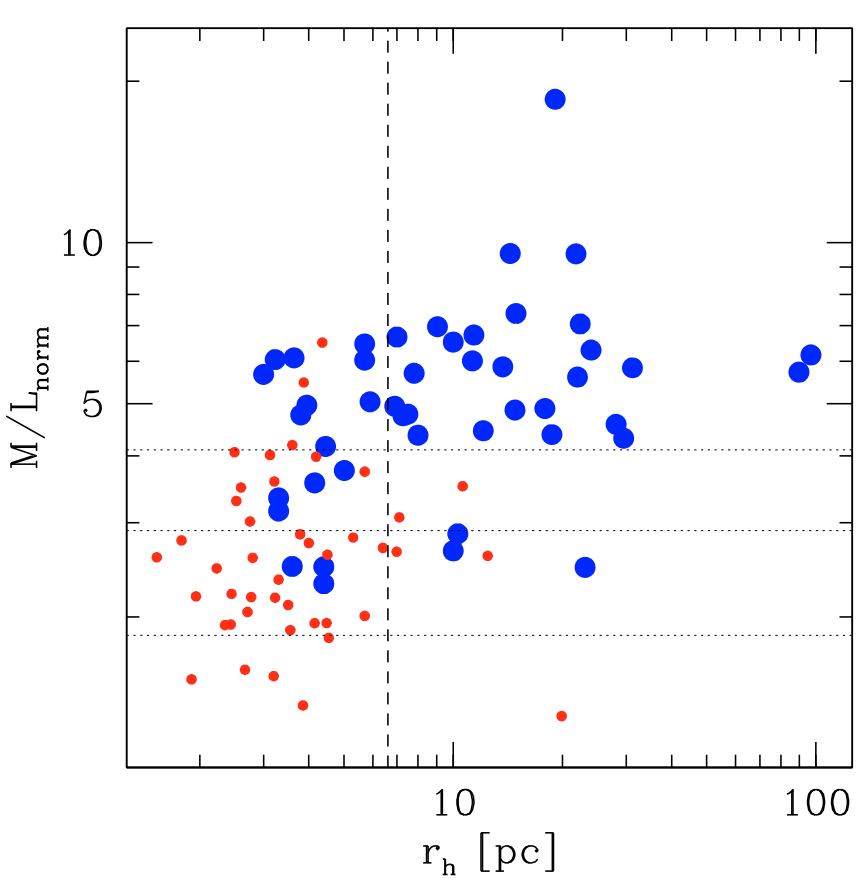

Fig. 13. Normalised $M / L$ ratio of the sample of compact stellar systems from Fig. 12 plotted against their effective half-light radius $r_{h}$ in pc. Small (red) dots indicate GCs, defined as compact stellar systems with $M<2 \times 10^{6} M_{\odot}$. Large (blue) dots are UCDs, defined as compact stellar systems with $M>2 \times 10^{6} M_{\odot}$. The vertical dashed line indicates the radius where the relaxation time is equal to one Hubble time.

to $(V-I)$ using the calibration relation of Kissler-Patig et al. (1998). In the plots we adopt a simple mass limit of $2 \times 10^{6} M_{\odot}$ to separate UCDs from GCs (see Fig. 12).

Zaritsky et al. (2006a,b, 2008) define the fundamental manifold relation as

$\log \left(r_{\mathrm{e}}\right)=2 \times \log (\sigma)-\log \left(I_{\mathrm{e}}\right)-\log \left(\Upsilon_{\mathrm{e}}^{f}\right)$.

In this formulation, $\log \left(\Upsilon_{\mathrm{e}}^{f}\right)$ is the effective mass-to-light ratio parametrized in terms of $\log (\sigma)$ and $\log \left(I_{\mathrm{e}}\right)$. That is, $\log \left(\Upsilon_{\mathrm{e}}^{f}\right)=\log \left(\Upsilon_{\mathrm{e}}^{f}\left(\log (\sigma), \log \left(I_{\mathrm{e}}\right)\right)\right.$. The parametrization is determined from a fit of dynamically derived $M / L$ ratio (using the virial theorem) as a function of $\log (\sigma)$ and $\log \left(I_{\mathrm{e}}\right)$ (see Zaritsky et al. 2008). It is clear that the exact functional shape of $\log \left(\Upsilon_{\mathrm{e}}^{f}\left(\log (\sigma), \log \left(I_{\mathrm{e}}\right)\right)\right.$ - and hence the location of the fundamental manifold - depends on which stellar systems are included in the fit. For example, the original formulation of the manifold (Zaritsky et al. 2006a) does not include the heavily dark matter dominated Local Group dwarf spheroidal galaxies in the fit (Zaritsky et al. 2006b; Simon \& Geha 2007). A revised formulation extending to the very large $M / L$ of the $\mathrm{dSphs}$ was presented in Zaritsky et al. (2008; see Table 1 of that paper).

We are interested in the link between UCDs and canonical galaxies on the one hand, and on the relation between UCDs and star clusters on the other hand. Therefore, we show in Fig. 14 the location of UCDs and GCs with respect to three different formulations of the fundamental manifold. The functional form of $\log \left(\Upsilon_{\mathrm{e}}^{f}\left(\log (\sigma), \log \left(I_{\mathrm{e}}\right)\right)\right.$ for these three representations is indicated in Table 4.

The first formulation does not include Local Group dwarf galaxies to the fit of $\log \left(\Upsilon_{\mathrm{e}}^{f}\left(\log (\sigma), \log \left(I_{\mathrm{e}}\right)\right)\right.$, nor UCDs and GCs. This is the original manifold version from Zaritsky et al. (2006a). It is intriguing that in this formulation, UCDs extend the fundamental manifold relation by more than a decade in $r_{\mathrm{e}}$, down to $r_{\mathrm{e}} \sim 5-7 \mathrm{pc}$ (note that $r_{\mathrm{e}}$ is shown in units of $\mathrm{kpc}$ in Fig. 14). Together with all other spheroids they follow a well defined linear function slightly inclined with respect to the original fundamental manifold, with a slope $0.92 \pm 0.01$. The fundamental manifold relation in this formulation breaks down only for the faintest dwarf spheroidal galaxies ( $M_{V}>-9$ mag) and for globular clusters. A possible interpretation of this is that for the faintest dwarf spheroidals, the dark matter halo is de-coupled from the baryons (see also Zaritsky et al. 2006b) such that the continous relation of baryon packing efficiency vs. galaxy scale breaks down. Another possibility is that the faintest dwarfs are out of dynamical equilibrium. This aspect is closely related to the discussion of the origin of dwarf satellite galaxies (dark-matter dominated cosmological substructure vs. tidal dwarf galaxy, see Kroupa et al. 2005 and Metz \& Kroupa 2007).

The second formulation includes all objects in Fig. 14 for the fitting $\log \left(\Upsilon_{\mathrm{e}}^{f}\left(\log (\sigma), \log \left(I_{\mathrm{e}}\right)\right)\right.$ (Zaritsky, private communication). Again, UCDs follow the manifold line, and only for $\log \left(r_{\mathrm{e}}\right) \lesssim-2.2$ they start to "bend down". Interestingly, at this radius also the transition between objects with relaxation times smaller and larger than a Hubble time occurs (Fig. 13). Globular clusters show a large scatter, and do clearly not align along the manifold.

The third formulation includes all objects for fitting except GCs and UCDs with $\log \left(r_{\mathrm{e}}\right)<-2.2$. This formulation hence excludes dynamically relaxed stellar systems from the fit. UCDs with $\log \left(r_{\mathrm{e}}\right)>-2.2$ align very well with the manifold, while globular clusters and smaller UCDs do not.

Summarizing, UCDs with $\log \left(r_{\mathrm{e}}\right)>-2.2\left(r_{\mathrm{e}} \gtrsim 7 \mathrm{pc}\right)$ appear to form a single family with larger stellar systems in the fundamental manifold. The location of most GCs is inconsistent with the fundamental manifold extrapolated from larger stellar systems. Dynamically un-relaxed stellar systems appear to form a single manifold, while the relaxed systems - due to their advanced dynamical evolution - scatter very broadly around it.

\section{Summary and conclusions}

In this paper we have analysed the internal dynamics of 23 ultracompact dwarf galaxies in the Fornax cluster. The analysis is based on high-resolution spectroscopy obtained with the FLAMES spectrograph at the VLT. Our targets cover an approximate mass range of $10^{6}<M<10^{8} M_{\odot}$ and a luminosity range $-10.4<M_{V}<-13.5 \mathrm{mag}$, overlapping the bright end of the 


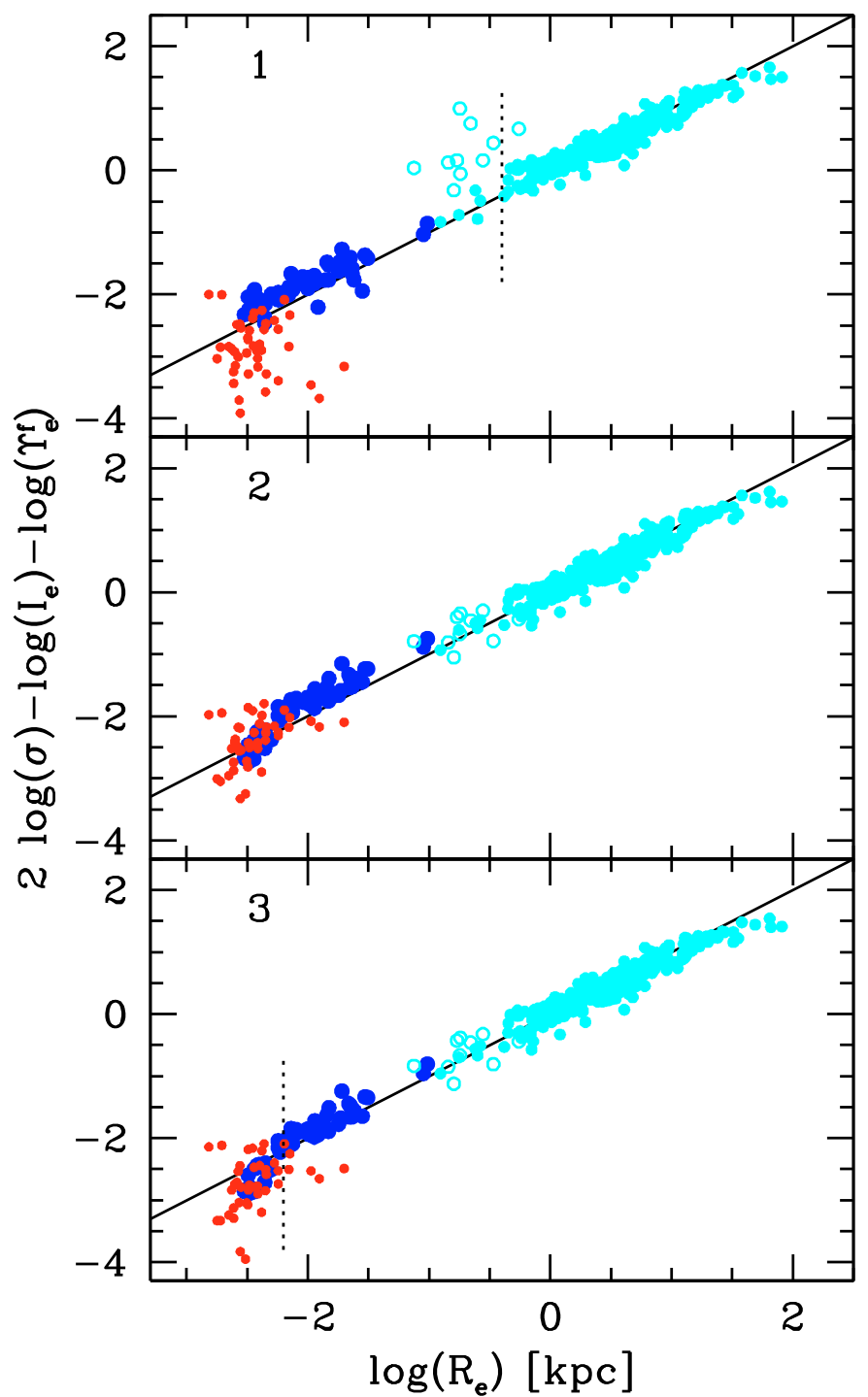

Fig. 14. The location of the compact stellar systems from Figs. 12 and 13 with respect to three different formulations of the "fundamental manifold" (Zaritsky et al. 2006a,b). Table 4 gives the fitting coefficients for the three formulations. Large blue dots are UCDs, small red dots are GCs. A global mass cut at $2 \times 10^{6} M_{\odot}$ is applied to separate UCDs from GCs. Cyan dots are large spheroids from the sample of Zaritsky et al. (2006a), with open cyan circles indicating faint Local Group dSphs with $M_{V}>-9$ mag (Zaritsky et al. 2006b, Simon $\&$ Geha 2007). Plot 1: this is the original formulation of the manifold (Zaritsky et al. 2006a), for which the fit of $\log \left(\Upsilon_{\mathrm{e}}^{f}\left(\log (\sigma), \log \left(I_{\mathrm{e}}\right)\right)\right.$ only includes large stellar spheroids with $\log \left(r_{\mathrm{e}}\right)>-0.4$, excluding the Local Group dSphs. Plot 2: the fit of $\log \left(\Upsilon_{\mathrm{e}}^{f}\left(\log (\sigma), \log \left(\mathrm{I}_{\mathrm{e}}\right)\right)\right.$ includes all objects in the plot (Zaritsky private communication). Plot 3: the fit of $\log \left(\Upsilon_{\mathrm{e}}^{f}\left(\log (\sigma), \log \left(I_{\mathrm{e}}\right)\right)\right.$ includes all objects in the plot except UCDs with $\log \left(r_{\mathrm{e}}\right)<-2.2$ and GCs (Zaritsky, private communication). See also Fig. 13 and text. The limiting $\log \left(r_{\mathrm{e}}\right)$ is marked by a dashed vertical tick.

globular cluster luminosity function. We also compare the dynamical properties of UCDs and GCs, and put them into the context of fundamental scaling relations defined for larger galaxies. We obtain the following results:

1. In the $M_{V}-\sigma$ plane, we find that UCDs with $M_{V}<$ -12 mag are consistent with the extrapolation of the Faber-Jackson relation for luminous elliptical galaxies. For $M_{V}>-12 \mathrm{mag}$, most objects are located closer to the extrapolation to brighter luminosities of the globular cluster $M_{V}-\sigma$ relation.

2. We derive dynamical $M / L$ ratios for those 15 of the 23 UCDs for which HST archival imaging is available, taking into account aperture effects in the spectroscopy (Hilker et al. 2007). Three out of the 15 UCDs have dynamical $M / L$ ratios too high to be explained by canonical stellar populations, but we do not find Fornax UCDs with $M / L$ ratios as extreme as found for some Virgo UCDs (Haşegan et al. 2005). At a given metallicity, Fornax UCDs have on average 30 to $40 \%$ lower $M / L$ ratios than Virgo UCDs.

3. We normalise the dynamical $M / L$ ratios of the 15 Fornax UCDs to solar metallicity, using predictions from stellar population models (Bruzual \& Charlot 2003; Maraston 2005). We find no significant correlation between normalised $M / L$ ratio and mass or relaxation time for our Fornax UCD sample. We do not find a dependence of normalised $\mathrm{M} / \mathrm{L}$ ratio on projected clustercentric distance.

4. We add our new measurements for 15 Fornax UCDs to the available data on $M / L$ ratios of compact stellar systems in the broader mass range $10^{4}<M<10^{8} M_{\odot}$. We include Galactic globular clusters and UCDs in Virgo, CenA and Fornax. We re-analyse dynamical mass estimates of UCDs in the Virgo cluster (Haşegan et al. 2005) and the CenA group (Rejkuba et al. 2007), using our modelling algorithm (Hilker et al. 2007) to correct for aperture effects in the spectroscopy. The corrections for the global velocity dispersion $\sigma$ are of the order of 5-10\%. We also provide previously unpublished $M$ and $M / L$ estimates for 5 CenA compact objects.

5. We find a clear break in the distribution of normalised $\mathrm{M} / \mathrm{L}$ ratios at a characteristic mass of $\simeq 2 \times 10^{6} M_{\odot}$, which roughly corresponds to a relaxation time of one Hubble time (see also Dabringhausen et al. 2008; Mieske \& Kroupa 2008). Objects more massive than this limit have normalised $\mathrm{M} / \mathrm{L}$ ratios twice as large as objects less massive than this limit. In this context we suggest to separate UCDs from GCs by a mass limit of $M \simeq 2 \times 10^{6} M_{\odot}$ (see also Haşegan et al. 2005). On average, the $M / L$ ratios of UCDs are $40 \%$ above the expectations for a $13 \mathrm{Gyr}$ stellar population with canonical IMF, while for GCs they are $40 \%$ below these expectations. We find that the $M / L$ ratios estimates of GCs are probably only weakly biased $(\sim 5 \%)$ by their dynamical evolution, indicating that stellar population models indeed over-predict $M / L$ ratios for compact stellar systems like GCs.

6. UCDs extend the "Fundamental Manifold" in its original formulation (Zaritsky et al. 2006a) by more than a decade in $r_{\mathrm{e}}$ down to $r_{\mathrm{e}} \sim 5-7 \mathrm{pc}$. In this formulation, neither the faintest dwarf spheroidals $\left(M_{V}>-9 \mathrm{mag}\right)$ nor GCs lie on the manifold. When using also GCs, UCDs and dSphs to define the shape of the FM, UCDs with $r_{\mathrm{e}} \gtrsim 7 \mathrm{pc}$ and dwarf spheroidals align along the manifold, while GCs and smaller UCDs do not. This characteristic scale of $r_{\mathrm{e}} \simeq 7 \mathrm{pc}$ also marks the transition between compact stellar systems with relaxation times below and above a Hubble time.

We suggest a defintion of UCDs as those compact stellar systems with $M \geq 2 \times 10^{6} M_{\odot}$ and $7 \lesssim r_{\mathrm{e}} / \mathrm{pc} \lesssim 100$. As such, UCDs are the smallest dynamically un-relaxed stellar systems. From their position in the "Fundamental Manifold" they can be considered the small-scale end of the galaxy sequence.

A key question about UCDs is whether they are of "cosmological" origin, hence related to compact low-mass dark matter halos. Their elevated $M / L$ ratios can be interpreted as marking the on-set of dark matter domination in small stellar 
systems. However, dark matter can hardly be detected directly, such that observational efforts need to be directed towards verifying/excluding alternative scenarios, such as a variation of the IMF in UCDs (Mieske \& Kroupa 2008). In parallel, theoretical studies regarding the dynamical evolution of compact stellar systems embedded in dark matter halos are needed for the mass-size regime of UCDs.

In this paper it has been found that Fornax UCDs have $30-40 \%$ lower dynamical $M / L$ ratios than Virgo UCDs. A possible explanation for this is that only Virgo UCDs have significant fractions of dark matter. This may be explained by the dominance of different UCD formation channels in Virgo and Fornax (Mieske et al. 2006). A simple way to test the possibility of different dark matter fractions is to determine the luminosity weighted ages of Fornax and Virgo UCDs. Younger ages in Fornax UCDs of $\sim 7$ Gyrs would naturally explain the $M / L$ ratio differences and imply similar dark matter fractions as in Virgo. Together with efforts to constrain the IMF shape in UCDs, such an observational study is the next logical step in understanding the puzzling nature of UCDs.

Acknowledgements. We warmly thank Dennis Zaritsky for providing us with updated fundamental manifold formulations. We also thank Matías Gomez for providing us with structural parameters for GCs in CenA.

\section{References}

Bassino, L. P., Muzzio, J. C., \& Rabolli, M. 1994, ApJ, 431, 634

Barmby, P., McLaughlin, D. E., Harris, W. E., Harris, G. L. H., \& Forbes, Duncan A. 2007, AJ, 133, 2764

Baumgardt, H., \& Makino, J. 2003, MNRAS, 340

Bekki, K., Couch, W. J., Drinkwater, M. J., \& Shioya, Y. 2003, MNRAS, 344, 399

Bruzual, G., \& Charlot, S. 2003, MNRAS, 344, 1000

Chabrier, G. 2003, PASP, 115, 763

Dabringhausen, J., Hilker, M., \& Kroupa, P. 2008, MNRAS, 395, 864

de Marchi, G. 1999, AJ, 117, 303

Drinkwater, M. J., Jones J. B., Gregg M. D., \& Phillipps, S. 2000, PASA, 17, 227

Drinkwater, M. J., Gregg, M. D., Hilker, M., et al. 2003, Nature, 423, 519

Dubath, P., \& Grillmair, C. J. 1997, A\&A, 321, 379

Dubath, P., Meylan, G., \& Mayor, M. 1992, ApJ, 400, 510

Evstigneeva, E. A., Gregg, M. D., Drinkwater, M. J., \& Hilker, M. 2007, AJ, 133,1722
Evstigneeva, E. A. Drinkwater, M. J., Peng, C. Y., et al. 2008, AJ, 136, 461 Fellhauer, M., \& Kroupa, P. 2002, MNRAS, 330, 642

Fellhauer, M., \& Kroupa, P. 2005, MNRAS, 359, 223

Fellhauer, M., \& Kroupa, P. 2006, MNRAS, 367, 1577

Ferguson, H. C. 1989, AJ, 98, 367

Freedman, W. L., Madore, B. F., Gibson, B. K., et al. 2001, ApJ, 553, 47

Gilmore, G., Wilkinson, M. I., Wyse, R. F. G., et al. 2007, ApJ, 663, 948

Goerdt, T., Moore, B., Kzantzidis, S., et al. 2008, MNRAS, 385, 2136

Haşegan, M., Jordán, A., Côté, P., et al. 2005, ApJ, 627, 203

Hilker, M., Infante, L., Vieira, G., Kissler-Patig, M., \& Richtler, T. 1999, A\&AS, 134,75

Hilker, M., Mieske, S., \& Infante, L. 2003, A\&A, 397, L9

Hilker, M., Baumgardt, H., Infante, L., et al. 2007, A\&A, 463, 119

Jordán, A. Blakeslee, J. P., Côté, P., et al. 2007, ApJS, 169, 213

Kissler-Patig, M. Brodie, Jean P., Schroder, L. L., et al. 1998, AJ, 115, 105

Kissler-Patig, M., Jordán, A., \& Bastian, N. 2006, A\&A, 448, 1031

Kroupa, P. 2001, MNRAS, 322, 231

Kroupa, P., Theis, C., \& Boily, C. M. 2005, A\&A, 431, 517

Lamers, H. J. G. L. M., Anders, P., \& de Grijs, R. 2006, A\&A, 452, 131

Maraston, C. 2005, MNRAS, 362, 799

Maraston, C., Bastian, N., Saglia, R. P., et al. 2004, A\&A, 416, 467

Martini, P., \& Ho, L. C. 2004, ApJ, 610, 233

McLaughlin, D. E., \& van der Marel, R. P. 2005, ApJS, 161, 304

Metz, M. \& Kroupa, P. 2007, MNRAS, 376, 387

Meylan, G., Sarajedini, A., Jablonka, P., Djorgovski, S. G., Bridges, T., \& Rich, R. M. 2001, AJ, 122, 830

Mieske, S., \& Kroupa, P. 2008, ApJ, 677, 276

Mieske, S., Hilker, M., \& Infante, L. 2002, A\&A, 383, 832

Mieske, S., Hilker, M., \& Infante, L. 2004, A\&A, 418, 445

Mieske, S., Hilker, M., Infante, L., \& Jordán, A. 2006, AJ, 131, 2442

Mieske, S., Hilker, M., Infante, L., \& Mendes de Oliveira, C. 2007a, A\&A, 463, 503

Mieske, S., Hilker, M., Jordán, A., Infante, L., \& Kissler-Patig, M. 2007b, A\&A, 472, 111

Milgrom, M. 1983, ApJ, 270, 365

Pasquini, L., Avila, G., Blecha, A., et al. 2002, ESO Messenger, 110, 1

Rejkuba, M. 2004, A\&A, 413, 903

Rejkuba, M., Dubath, P., Minniti, D., \& Meylan, G. 2007, A\&A, 469, 147

Richtler, T. Dirsch, B., Gebhardt, K., et al. 2004, AJ, 127, 2094

Richtler, T., Schubert, Y., Hilker, M., et al. 2008, A\&A, 478L, 23

Simon, J., \& Geha, M. 2007, ApJ, 670, 313

Spitzer, L. J. 1987 (Princeton, NJ: Princeton University Press), 191

Spitzer, L. J., \& Hart, M. H. 1971, ApJ, 164, 399

Tonry, J. L., Dressler, A., Blakeslee, J. P. et al. 2001, ApJ, 546, 681

van Loon, J., van Leeuwen, F., Smalley, B., et al. 2007, MNRAS, 382, 1353

Zaritsky, D., Gonzales, A., \& Zabludoff, A. 2006a, ApJ, 638, 725

Zaritsky, D., Gonzales, A., \& Zabludoff, A. 2006b, ApJ, 642L, 37

Zaritsky, D., Gonzales, A., \& Zabludoff, A. 2008, ApJ, submitted, [arXiv:0711.2071] 
Table 5. This table gives masses, $M / L_{V}$ ratios, metallicities, $M / L_{V}$ ratios normalised to solar metallicity, half-light radii and velocity dispersions $\sigma$ for the compact stellar systems from Fig. 12 to Fig. 14. The table is ordered by descending mass. Sources are this paper ${ }^{a}$, Hilker et al. (2007) ${ }^{b}$, Rejkuba et al. (2007) $)^{c}$, Haşegan et al. (2005) $)^{d}$, Evstigneeva et al. (2007) $)^{e}$, McLaughlin \& van der Marel (2005) $)^{f}$, Meylan et al. (2001) $)^{g}$, Barmby et al. $(2007)^{h}$, de Marchi (1999) $)^{i}$. For galactic sources (ID MW...; McLaughlin \& van der Marel 2005), the quoted $\sigma$ measurements are close to central values. For extragalactic sources, $\sigma$ is the global velocity dispersion, corrected for spectroscopy aperture losses (see text and Hilker et al. 2007).

\begin{tabular}{|c|c|c|c|c|c|c|}
\hline ID & Mass $\left[10^{6} M_{\odot}\right]$ & $M / L$ & {$[\mathrm{Fe} / \mathrm{H}][\mathrm{dex}]$} & $M / L_{\text {norm }}$ & $r_{\mathrm{h}}[\mathrm{pc}]$ & $\sigma\left[\mathrm{km} \mathrm{s}^{-1}\right]$ \\
\hline$\overline{\mathrm{F}-19^{a}}$ & $93.6(14.0)$ & $4.69(0.70)$ & -0.4 & $\overline{5.73(1.03)}$ & 89.7 & 22.8 \\
\hline $\mathrm{VUCD}^{e}$ & $88.3(22.0)$ & $4.39(1.10)$ & -0.7 & $6.17(1.61)$ & 96.8 & 27.2 \\
\hline $\mathrm{VUCD}^{e}$ & $40.0(5.9)$ & $4.35(0.64)$ & 0.0 & $4.38(0.83)$ & 18.7 & 35.8 \\
\hline $\mathrm{UCD} 1^{b}$ & 32.1 (3.9) & $4.99(0.60)$ & -0.7 & $7.05(1.02)$ & 22.4 & 27.1 \\
\hline $\mathrm{S} 417^{d}$ & $29.5(6.0)$ & $6.68(1.40)$ & -0.7 & $9.54(2.08)$ & 14.4 & 29.8 \\
\hline $\mathrm{VUCD}^{e}$ & $29.1(4.3)$ & $4.02(0.60)$ & -0.4 & $4.90(0.88)$ & 17.9 & 26.4 \\
\hline $\mathrm{VUCD}^{e}$ & $28.2(4.7)$ & $4.11(0.69)$ & -0.8 & $6.01(1.11)$ & 11.3 & 32.2 \\
\hline $\mathrm{F}-24^{a}$ & $24.5(7.8)$ & $3.44(1.10)$ & -0.4 & $4.31(1.43)$ & 29.5 & 21.4 \\
\hline $\mathrm{VUCD}^{e}$ & $24.3(6.3)$ & $3.45(0.89)$ & -1.0 & $5.61(1.49)$ & 22.0 & 21.3 \\
\hline S999 ${ }^{d}$ & $23.4(4.3)$ & $10.2(1.90)$ & -1.4 & $18.5(3.5)$ & 19.1 & 22.7 \\
\hline $\mathrm{S} 928^{d}$ & $19.3(4.5)$ & $5.32(1.20)$ & -1.3 & $9.52(2.25)$ & 21.8 & 19.1 \\
\hline $\mathrm{UCD}^{b}$ & $18.0(4.5)$ & $3.37(0.85)$ & -1.2 & $5.84(1.50)$ & 31.2 & 18.7 \\
\hline $\mathrm{VUCD}^{e}$ & $17.7(5.5)$ & $3.02(0.94)$ & -1.0 & $4.87(1.54)$ & 14.8 & 22.3 \\
\hline $\mathrm{F}-1^{a}$ & $16.2(3.8)$ & $2.45(0.58)$ & 0.0 & $2.48(0.66)$ & 23.1 & 18.7 \\
\hline $\mathrm{S} 490^{d}$ & $14.5(0.3)$ & $6.81(0.15)$ & 0.2 & $6.09(0.82)$ & 3.6 & 41.6 \\
\hline $\mathrm{F}-9^{a}$ & $14.1(3.6)$ & $4.72(1.20)$ & -0.8 & $6.96(1.85)$ & 9.1 & 25.7 \\
\hline $\mathrm{F}-5^{a}$ & $13.7(2.4)$ & $3.16(0.55)$ & -0.3 & $3.75(0.76)$ & 5.0 & 34.5 \\
\hline $\mathrm{F}-6^{a}$ & $12.5(2.4)$ & $5.32(1.00)$ & 0.2 & $4.75(1.09)$ & 7.3 & 27.3 \\
\hline $\mathrm{HCH} 99-18^{c}$ & $11.2(4.3)$ & $3.68(1.40)$ & -1.0 & $5.86(2.26)$ & 13.7 & 18.7 \\
\hline $\mathrm{F}-7^{a}$ & 10.5 (1.4) & $4.21(0.57)$ & -1.3 & 7.37 (1.07) & 14.9 & 20.1 \\
\hline $\mathrm{S} 314^{d}$ & $9.1(1.3)$ & $4.63(0.68)$ & -0.5 & $6.05(1.04)$ & 3.2 & 34.9 \\
\hline $\mathrm{F}-12^{a}$ & $8.3(2.9)$ & $2.36(0.83)$ & -0.4 & $2.86(1.05)$ & 10.3 & 22.9 \\
\hline $\mathrm{G} 1^{g}$ & $7.2(1.2)$ & $3.6(0.60)$ & -1.0 & $5.67(1.01)$ & $3.0^{h}$ & 25.0 \\
\hline $\mathrm{HGHH} 92-\mathrm{C} 1^{c}$ & $6.8(1.7)$ & $3.67(0.90)$ & -1.2 & $6.30(1.58)$ & 24.0 & 11.1 \\
\hline $\mathrm{HGHH} 92-\mathrm{C} 23^{c}$ & $6.6(2.2)$ & $1.68(0.55)$ & -1.5 & $3.16(1.04)$ & 3.3 & 29.5 \\
\hline $\mathrm{HGHH} 92-\mathrm{C}^{c}$ & $6.3(2.2)$ & $2.68(0.95)$ & -1.3 & $4.78(1.71)$ & 7.5 & 19.1 \\
\hline $\mathrm{F}-17^{a}$ & $6.3(1.6)$ & $2.22(0.55)$ & -0.8 & $3.34(0.86)$ & 3.3 & 28.5 \\
\hline $\mathrm{F}-11^{a}$ & $5.7(3.7)$ & $1.64(1.10)$ & -0.9 & $2.49(1.63)$ & 3.6 & 26.2 \\
\hline $\mathrm{HCH} 99-15^{c}$ & $5.6(1.7)$ & $3.11(0.95)$ & -1.0 & $5.04(1.57)$ & 5.9 & 20.5 \\
\hline $\mathrm{F}-34^{a}$ & $5.5(1.3)$ & $3.17(0.74)$ & -0.9 & $4.97(1.20)$ & 4.0 & 24.6 \\
\hline $\mathrm{F}-22^{a}$ & $5.3(1.0)$ & $2.13(0.39)$ & -0.4 & $2.66(0.55)$ & 10.0 & 22.8 \\
\hline $\mathrm{HGHH} 92-\mathrm{C} 11^{c}$ & $5.3(1.9)$ & $4.45(1.60)$ & -0.5 & $5.70(2.18)$ & 7.8 & 17.1 \\
\hline $\mathrm{HGHH} 92-\mathrm{C} 17^{c}$ & $5.1(1.7)$ & $3.39(1.10)$ & -1.3 & $6.03(1.98)$ & 5.7 & 19.8 \\
\hline VHH81-C5 ${ }^{c}$ & $5.0(1.2)$ & $3.39(0.80)$ & -1.6 & $6.52(1.57)$ & 10.0 & 14.8 \\
\hline $\mathrm{HGHH} 92-\mathrm{C} 21^{c}$ & $4.8(1.7)$ & $3.87(1.40)$ & -1.2 & $6.66(2.35)$ & 7.0 & 17.2 \\
\hline $\mathrm{H} 8005^{d}$ & $4.8(2.5)$ & $2.61(1.40)$ & -1.3 & $4.58(2.38)$ & 28.1 & 8.5 \\
\hline HCH99- $2^{c}$ & $4.2(1.6)$ & $3.62(1.40)$ & -1.5 & $6.72(2.62)$ & 11.4 & 12.5 \\
\hline $\mathrm{F}-53^{a}$ & $3.9(1.0)$ & $2.66(0.69)$ & -0.9 & $4.16(1.11)$ & 4.4 & 19.6 \\
\hline $\mathrm{HGHH} 92-\mathrm{C6}^{c}$ & $3.6(0.9)$ & $1.60(0.40)$ & -0.9 & $2.48(0.64)$ & 4.4 & 19.0 \\
\hline $\mathrm{F}-51^{a}$ & $3.5(0.9)$ & $2.38(0.62)$ & -0.8 & $3.56(0.96)$ & 4.2 & 20.1 \\
\hline $\mathrm{HGHH} 92-\mathrm{C} 29^{c}$ & $3.3(1.1)$ & $3.51(1.20)$ & -0.7 & $4.95(1.74)$ & 6.9 & 14.5 \\
\hline$\omega \operatorname{Cen}^{c}$ & $3.0(0.5)$ & $2.40(0.40)$ & -1.6 & $4.61(0.80)$ & $8.0^{i}$ & 16.0 \\
\hline $\mathrm{HGHH} 92-\mathrm{C} 22^{c}$ & $2.6(0.8)$ & $2.76(0.85)$ & -1.2 & $4.77(1.49)$ & 3.8 & 17.2 \\
\hline VHH81-C3 ${ }^{c}$ & $2.4(0.7)$ & $1.68(0.50)$ & -0.6 & $2.31(0.72)$ & 4.4 & 15.2 \\
\hline HCH99-16 & $2.0(0.7)$ & $2.16(0.80)$ & -1.9 & $4.45(1.67)$ & 12.1 & 8.4 \\
\hline $\mathrm{HGHH} 92-\mathrm{C} 44^{c}$ & $1.9(0.6)$ & $3.38(1.10)$ & -1.6 & $6.47(2.13)$ & 5.7 & 12.1 \\
\hline HGHH92-C36=R01-113 ${ }^{c}$ & $1.8(0.6)$ & $2.25(0.75)$ & -1.5 & $4.19(1.41)$ & 3.6 & 14.7 \\
\hline $\mathrm{HCH} 99-21^{c}$ & $1.6(0.9)$ & $1.48(0.85)$ & -2.0 & $3.07(1.77)$ & 7.1 & 9.7 \\
\hline HНH86-C18 ${ }^{c}$ & $1.6(0.5)$ & $0.95(0.30)$ & -1.1 & $1.55(0.50)$ & 3.2 & 14.7 \\
\hline MW-NGC6715 ${ }^{f}$ & $1.5(0.6)$ & $1.41(0.58)$ & -1.6 & $2.69(1.11)$ & 6.4 & 14.2 \\
\hline $\mathrm{F}-59^{a}$ & $1.3(0.6)$ & $0.94(0.43)$ & -2.1 & $2.01(0.93)$ & 5.7 & 9.8 \\
\hline HHH86-C15=R01-226 ${ }^{c}$ & $1.3(0.5)$ & $1.93(0.75)$ & -0.8 & $2.81(1.11)$ & 5.3 & 10.1 \\
\hline $\mathrm{HGHH} 92-\mathrm{C} 41^{c}$ & $1.2(0.4)$ & $1.87(0.60)$ & -0.7 & $2.61(0.87)$ & 4.5 & 10.7 \\
\hline $\mathrm{R} 01-223^{c}$ & $1.1(0.4)$ & $2.08(0.75)$ & -1.1 & $3.49(1.27)$ & 2.6 & 13.7 \\
\hline HHH86-C38=R01-123 ${ }^{c}$ & $1.1(0.4)$ & $1.48(0.50)$ & -1.2 & $2.58(0.88)$ & 2.8 & 13.6 \\
\hline
\end{tabular}


Table 5. continued.

\begin{tabular}{|c|c|c|c|c|c|c|}
\hline ID & Mass $\left[10^{6} M_{\odot}\right]$ & $M / L$ & {$[\mathrm{Fe} / \mathrm{H}][\mathrm{dex}]$} & $M / L_{\text {norm }}$ & $r_{\mathrm{h}}[\mathrm{pc}]$ & $\sigma\left[\mathrm{km} \mathrm{s}^{-1}\right]$ \\
\hline HGHH92-C37=R01-116 & $1.1(0.4)$ & $1.49(0.50)$ & -1.0 & $2.35(0.80)$ & 3.3 & 12.0 \\
\hline MW-NGC6441 ${ }^{f}$ & $0.91(0.47)$ & $1.65(0.85)$ & -0.5 & $2.19(1.14)$ & 2.0 & 18.0 \\
\hline $\mathrm{R} 01-261^{c}$ & $0.87(0.32)$ & $0.96(0.35)$ & -1.0 & $1.53(0.57)$ & 1.9 & 14.2 \\
\hline MW-NGC6388 ${ }^{f}$ & $0.79(0.37)$ & $1.89(0.90)$ & -0.6 & $2.58(1.24)$ & 1.5 & 18.9 \\
\hline MW-NGC5824 ${ }^{f}$ & $0.68(0.28)$ & $1.96(0.82)$ & -1.9 & $3.98(1.68)$ & 4.2 & 11.6 \\
\hline MW-NGC104 ${ }^{f}$ & $0.64(0.26)$ & $1.33(0.54)$ & -0.8 & $1.95(0.80)$ & 4.2 & 11.5 \\
\hline MW-NGC2808 ${ }^{f}$ & $0.61(0.27)$ & $1.46(0.64)$ & -1.2 & $2.47(1.09)$ & 2.2 & 13.4 \\
\hline MW-NGC6656 ${ }^{f}$ & $0.40(0.19)$ & $2.07(0.99)$ & -1.6 & $4.01(1.92)$ & 3.1 & 9.0 \\
\hline MW-NGC6864 ${ }^{f}$ & $0.40(0.17)$ & $1.78(0.75)$ & -1.2 & $3.02(1.27)$ & 2.8 & 10.3 \\
\hline MW-NGC6402f & $0.38(0.21)$ & $1.16(0.64)$ & -1.4 & $2.11(1.16)$ & 3.5 & 8.2 \\
\hline MW-NGC7089 ${ }^{f}$ & $0.36(0.15)$ & $0.98(0.40)$ & -1.6 & $1.89(0.78)$ & 3.6 & 8.2 \\
\hline MW-NGC6205 ${ }^{f}$ & $0.29(0.12)$ & $1.51(0.62)$ & -1.5 & $2.85(1.19)$ & 3.8 & 7.1 \\
\hline MW-NGC2419 $f$ & $0.29(0.11)$ & $0.61(0.24)$ & -2.1 & $1.31(0.53)$ & 19.9 & 3.0 \\
\hline MW-NGC5272 $f$ & $0.28(0.11)$ & $1.39(0.57)$ & -1.6 & $2.65(1.08)$ & 7.0 & 5.6 \\
\hline MW-NGC1851 ${ }^{f}$ & $0.26(0.10)$ & $1.61(0.65)$ & -1.2 & $2.78(1.12)$ & 1.8 & 10.4 \\
\hline MW-NGC5286 $f$ & $0.24(0.11)$ & $0.99(0.44)$ & -1.7 & $1.93(0.87)$ & 2.4 & 8.0 \\
\hline MW-NGC5904 ${ }^{f}$ & $0.18(0.07)$ & $0.78(0.32)$ & -1.3 & $1.37(0.56)$ & 3.9 & 5.7 \\
\hline MW-NGC6254 ${ }^{f}$ & $0.17(0.07)$ & $2.16(0.98)$ & -1.5 & $4.06(1.84)$ & 2.5 & 6.6 \\
\hline MW-NGC3201 ${ }^{f}$ & $0.17(0.07)$ & $2.87(1.20)$ & -1.6 & $5.48(2.30)$ & 3.9 & 5.2 \\
\hline MW-NGC6809 $f$ & $0.17(0.07)$ & $3.23(1.30)$ & -1.8 & $6.51(2.65)$ & 4.4 & 4.9 \\
\hline MW-NGC5694 ${ }^{f}$ & $0.16(0.06)$ & $1.35(0.54)$ & -1.9 & $2.75(1.11)$ & 4.0 & 5.5 \\
\hline MW-NGC6341 ${ }^{f}$ & $0.12(0.05)$ & $0.88(0.37)$ & -2.3 & $1.94(0.83)$ & 2.4 & 5.9 \\
\hline MW-NGC1904 ${ }^{f}$ & $0.096(0.039)$ & $1.16(0.47)$ & -1.6 & $2.21(0.90)$ & 2.5 & 5.2 \\
\hline MW-NGC6171 ${ }^{f}$ & $0.084(0.040)$ & $2.20(1.00)$ & -1.0 & $3.58(1.71)$ & 3.2 & 4.1 \\
\hline MW-NGC6218 $f$ & $0.084(0.035)$ & $1.77(0.74)$ & -1.5 & $3.29(1.39)$ & 2.5 & 4.5 \\
\hline MW-NGC6779 $f$ & $0.081(0.035)$ & $1.05(0.45)$ & -1.9 & $2.17(0.94)$ & 3.2 & 4.0 \\
\hline MW-NGC6712f & $0.080(0.039)$ & $0.99(0.48)$ & -1.0 & $1.59(0.77)$ & 2.7 & 4.3 \\
\hline MW-NGC288 ${ }^{f}$ & $0.078(0.032)$ & $2.15(0.89)$ & -1.2 & $3.74(1.56)$ & 5.7 & 2.91 \\
\hline MW-NGC6121 ${ }^{f}$ & $0.073(0.035)$ & $1.27(0.61)$ & -1.2 & $2.18(1.06)$ & 2.8 & 4.2 \\
\hline MW-NGC6362 ${ }^{f}$ & $0.058(0.024)$ & $1.16(0.47)$ & -1.0 & $1.83(0.75)$ & 4.5 & 2.8 \\
\hline MW-NGC5466 ${ }^{f}$ & $0.049(0.020)$ & $1.61(0.67)$ & -2.2 & $3.51(1.49)$ & 10.6 & 1.7 \\
\hline MW-NGC4590 $f$ & $0.044(0.018)$ & $0.92(0.37)$ & -2.1 & $1.95(0.80)$ & 4.5 & 2.5 \\
\hline MW-NGC5053 ${ }^{f}$ & $0.038(0.016)$ & $1.18(0.48)$ & -2.3 & $2.60(1.09)$ & 12.4 & 1.4 \\
\hline MW-NGC4147f & $0.025(0.010)$ & $1.01(0.42)$ & -1.8 & $2.04(0.86)$ & 2.7 & 2.6 \\
\hline MW-NGC6366 $f$ & $0.00807(0.0044)$ & $0.30(0.17)$ & -0.8 & $0.45(0.25)$ & 3.1 & 1.3 \\
\hline
\end{tabular}

